\title{
The mean infrared emission of Sagittarius $A^{*}$
}

\author{
R. Schödel ${ }^{1}$, M. R. Morris ${ }^{2}$, K. Muzic ${ }^{3}$, A. Alberdi ${ }^{1}$, L. Meyer $^{2}$, A. Eckart ${ }^{4}$, and D. Y. Gezari ${ }^{5}$ \\ 1 Instituto de Astrofísica de Andalucía (CSIC), Glorieta de la Astronomía s/n, 18008 Granada, Spain \\ e-mail: rainer@iaa.es \\ 2 UCLA Division of Astronomy and Astrophysics, Physics and Astronomy Building, 430 Portola Plaza, Box 951547, Los Angeles, \\ CA 90095-1547, USA \\ 3 Department of Astronomy and Astrophysics, University of Toronto, 50 St. George Street, M5S 3H4 Toronto ON, Canada \\ ${ }^{4}$ I. Physikalisches Institut, Universität zu Köln, Zülpicher Str. 77, 50937 Köln, Germany \\ 5 NASA/Goddard Space Flight Center, Code 667, Greenbelt, MD 20771, USA \\ Received 31 March 2011 / Accepted 20 June 2011
}

\section{ABSTRACT}

\begin{abstract}
Context. The massive black hole at the center of the Milky Way, Sagittarius A* (Sgr A*) is, in relative terms, the weakest accreting black hole accessible to observations. It has inspired the theoretical models of radiatively inefficient accretion. Unfortunately, our knowledge of the mean SED and source structure of Sgr A* is very limited owing to numerous observational difficulties. At the moment, the mean SED of Sgr A* is only known reliably in the radio to $\mathrm{mm}$ regimes.

Aims. The goal of this paper is to provide constraints on the mean emission from Sgr A* in the near-to-mid infrared.

Methods. Sensitive images of the surroundings of Sgr A* at $8.6 \mu \mathrm{m}, 4.8 \mu \mathrm{m}$, and $3.8 \mu \mathrm{m}$ were produced by combining large quantities of imaging data. Images were produced for several observing epochs. Excellent imaging quality was reached in the MIR by using speckle imaging combined with holographic image reconstruction, a novel technique for this kind of data.

Results. No counterpart of Sgr A* is detected at $8.6 \mu \mathrm{m}$. At this wavelength, Sgr A* is located atop a dust ridge, which considerably complicates the search for a potential point source. An observed $3 \sigma$ upper limit of $\sim 10 \mathrm{mJy}$ is estimated for the emission of Sgr A* at $8.6 \mu \mathrm{m}$, a tighter limit at this wavelength than in previous work. The de-reddened $3 \sigma$ upper limit, including the uncertainty of the extinction correction, is $\sim 84 \mathrm{mJy}$. Based on the available data, it is argued that, with currently available instruments, Sgr A* cannot be detected in the MIR, not even during flares. At $4.8 \mu \mathrm{m}$ and $3.8 \mu \mathrm{m}$, on the other hand, Sgr $\mathrm{A}^{*}$ is detected at all times, at least when considering timescales of a few up to $13 \mathrm{~min}$. We derive well-defined time-averaged, de-reddened flux densities of $3.8 \pm 1.3 \mathrm{mJy}$ at $4.8 \mu \mathrm{m}$ and $5.0 \pm 0.6 \mathrm{mJy}$ at $3.8 \mu \mathrm{m}$. Observations with NIRC2/Keck and $\mathrm{NaCo} / \mathrm{VLT}$ from the literature provide good evidence that Sgr A* also has a fairly well-defined de-reddened mean flux of $0.5-2.5 \mathrm{mJy}$ at wavelengths of $2.1-2.2 \mu \mathrm{m}$.

Conclusions. We present well-constrained anchor points for the SED of Sgr A* on the high-frequency side of the Terahertz peak. The new data are in general agreement with published theoretical SEDs of the mean emission from Sgr A*, but we expect them to have an appreciable impact on the model parameters in future theoretical work.
\end{abstract}

Key words. black hole physics - instrumentation: high angular resolution - instrumentation: adaptive optics - Galaxy: center

\section{Introduction}

Sagittarius A* $\left(\operatorname{Sgr} A^{*}\right)$ is the name of the electromagnetic source related to the massive black hole at the center of the Milky Way. It is located at a distance of about $8 \mathrm{kpc}$ and has a mass of roughly 4 million solar masses (e.g., Ghez et al. 2008; Gillessen et al. 2009). That its bolometric luminosity amounts to only about $10^{-9}$ times its Eddington luminosity makes Sgr A* in relative terms - the weakest accreting black hole currently accessible to observation. It is therefore of great interest for developing and testing theories of accretion and emission in the weak accretion limit. Sgr A* was key to the development of theories of radiatively inefficient accretion, such as the ADAF, ADIOS, jet-ADAF, RIAF, CDAF, or similar models (see, e.g., review by Quataert 2003). An essential feature of all these models is their success in explaining the extremely low efficiency of converting accretion power into electromagnetic radiation, which is for weakly accreting sources like Sgr A* many orders of magnitude lower than in the case of the standard thin accretion disk. See the recent reviews by Melia \& Falcke (2001) and Genzel et al. (2010) for references and details.

Observationally, $\mathrm{Sgr}^{*} *$ is a difficult target for a combination of causes. In the regime of gamma and X-rays, the target is relatively faint, angular resolution is comparatively low, and confusion with other point or extended sources can be strong. Strong extinction makes observations in the optical to ultraviolet/soft X-ray domains all but impossible. In the near-infrared, Sgr A* is faint and confused with the extremely dense surrounding star cluster. In the mid-and far-infrared, it has so far eluded detection because of the high thermal background and confusion with interstellar dust emission. Sensitive observations with high angular resolution are only just now becoming possible in the Terahertz regime, but are technically highly challenging. At centimeter wavelengths, finally, the source is broadened significantly by interstellar scattering. The intrinsic source structure can be resolved with very long baseline interferometry at $\mathrm{mm}$ to submm wavelengths (e.g., Doeleman et al. 2008), but there are still large technical difficulties. All these factors together mean that even almost four decades after its detection (Balick \& Brown 1974), we still do not know the intrinsic source structure of $\operatorname{Sgr} \mathrm{A}^{*}$, and there are still gaps of several orders of magnitude in its observed spectral energy distribution (SED).

An additional aspect that must be taken into account when examining $\mathrm{Sgr}^{*} *$ is that the source is highly variable at wavelengths shorter than a few millimeters. Particularly in the nearinfrared (NIR) and X-ray domains, Sgr A* shows episodes of 
significantly increased emission. These so-called flares last on the order of $100 \mathrm{~min}$, with significant substructure (subflares) on timescales of $\sim 20 \mathrm{~min}$. The rise and fall times of flares and subflares can be as short as a few minutes (see, e.g., Baganoff et al. 2001; Genzel et al. 2003; Ghez et al. 2004; Eckart et al. 2008a; Meyer et al. 2008; Porquet et al. 2008; Yusef-Zadeh et al. 2008; Eckart et al. 2009; Yusef-Zadeh et al. 2009; Dodds-Eden et al. 2011). Most of the time, however, Sgr A* is very weak at both $\mathrm{X}$-rays and in the near-infrared. Both infrared observational and theoretical efforts have been focused almost exclusively on the flares so far. An exception are the works by Do et al. (2009) and Dodds-Eden et al. (2011), which are not biased toward flare emission and show that Sgr A* is continuously variable at wavelengths of $2.1-2.2 \mu \mathrm{m}$ (in the so-called $K$-band). While the analysis of the short-timescale variability is of great interest for the emission and accretion processes or even potentially for tests of general relativity (e.g., Broderick \& Loeb 2006; Meyer et al. 2006; Paumard et al. 2008; Zamaninasab et al. 2011), it is principally the mean, time-averaged emission that is of interest for models of the accretion/outflow processes that give rise to the overall SED of Sgr A* (e.g., Yuan et al. 2002; Mościbrodzka et al. 2009). Since there may be up to a few bright flares per day, these timescales range from one to several days. In this work, we focus on the mean emission of Sgr A* in the infrared regime.

The goal of this paper is to improve on available measurements of the mean emission from $\operatorname{Sgr~A}^{*}$, and in particular, to tighten the constraints at $8.6 \mu \mathrm{m}$ and to measure its mean fluxdensity at 3.8 and $4.8 \mu \mathrm{m}$.

Sgr A* is located within the so-called mini-spiral, a prominent feature of the interstellar medium in the central parsec of the Galactic center (GC) that is bright at mid-infrared (MIR) wavelengths, probably due to emission from warm dust (see, e.g., reviews by Morris \& Serabyn 1996; Mezger et al. 1996; Genzel et al. 2010). High angular resolution is therefore required to separate Sgr A* from the surrounding complex ISM emission. Stolovy et al. (1996) reported that Sgr A* sits on a ridge of emission, from their deconvolved images. Later observations showed the ridge much more clearly and that there is no obvious point-source corresponding to $\mathrm{Sgr} \mathrm{A}^{*}$, which could be separated from the ridge (Schödel et al. 2007). All attempts to find a MIR counterpart of Sgr A* have been unsuccessful so far, even during NIR flares (most recent work by Eckart et al. 2006a; Schödel et al. 2007; Dodds-Eden et al. 2009). Extinctioncorrected $3 \sigma$ upper limits on Sgr A* so far in the MIR are reported as $64 \mathrm{mJy}$ at $8.59 \mu \mathrm{m}$ (Schödel et al. 2007) and $57 \mathrm{mJy}$ at $11.88 \mu \mathrm{m}$ (Dodds-Eden et al. 2009) $)^{1}$.

As concerns the $M^{\prime}$-band, we are only aware of two publications reporting on $\mathrm{Sgr} \mathrm{A}^{*}$ at $4.8 \mu \mathrm{m}$. Clénet et al. (2004) reported on $M^{\prime}$ observations of a $\mathrm{Sgr} \mathrm{A}^{*}$ counterpart with $\mathrm{NaCo} / \mathrm{VLT}$ at two epochs, but it is not clear whether they could distinguish clearly between $\operatorname{Sgr}^{*}$ and a nearby dust-blob (Ghez et al. 2005). Hornstein et al. (2007) report the detection of a variable Sgr A* counterpart with NIRC2/Keck in $M_{\mathrm{s}}{ }^{2}$ during one observational epoch with NIRC2/Keck. In this paper we do not intend to resolve the variability of $\mathrm{Sgr} \mathrm{A}^{*}$ on short timescales, but infer its mean luminosity in $M^{\prime}$ during a large number of epochs and on deep images that average several observing runs.

\footnotetext{
1 These upper limits do not include the uncertainty of the extinction correction. The extinction assumed by Schödel et al. (2007) is probably under-estimated.

2 Within the accuracy required for this work, we can neglect the difference between $\mathrm{NaCo} M^{\prime}, \lambda_{\text {central }}=4.78 \mu \mathrm{m}$ and $\Delta \lambda=0.59$, and NIRC2 $M_{\mathrm{s}}, \lambda_{\text {central }}=4.67 \mu \mathrm{m}$ and $\Delta \lambda=0.24$.
}

High sensitivity and accuracy at $8.6 \mu \mathrm{m}$ is reached in this work by using large quantities of imaging data from many observing epochs. Additionally, the novel application of the speckle holography technique to MIR imaging data delivers high-Strehl images for all epochs and makes deconvolution unnecessary.

The properties of ISM and stars from imaging of a larger region between $3.8 \mu \mathrm{m}$ and $8.6 \mu \mathrm{m}$, about $20^{\prime \prime} \times 20^{\prime \prime}$ around Sgr A*, are treated in an upcoming companion paper by Schödel \& Morris.

The following section describes the data used for this work and their reduction. We then briefly discuss the ISM emission near Sgr A* and subsequently derive an upper limit on the emission from $\mathrm{Sgr}^{*}$ at $8.6 \mu \mathrm{m}$. Measurements of the mean flux density of Sgr A* in the $M-, L$-, and $K$-bands are presented in Sects. $3.3,3.4$, and $3.5^{3}$. We proceed to discuss the newly derived data on the mean infrared emission from $\mathrm{Sgr} \mathrm{A}^{*}$ within the context of models for its overall SED. In the subsequent section, we argue that it is rather improbable to detect either the mean emission or flares from Sgr A* in the MIR by standard imaging with current telescopes and instruments. The final section summarizes our conclusions.

\section{Observations and data reduction}

\subsection{Observations with VISIR/VLT at $8.6 \mu \mathrm{m}$}

All mid-infrared (MIR) imaging data used in this work were acquired with the MIR camera VISIR at the ESO VLT (Lagage et al. 2004). The PAH 1 filter was used, with a central wavelength of $8.59 \mu \mathrm{m}$ and a half-band width of $0.42 \mu \mathrm{m}$. Data used in this work are from 5 June 2006, 1/3/5/6/8 April 2007, 22/23 May 2007, and 20/21/23/24 July 2007 (all dates given in UTC) ${ }^{4}$. The pixel scale was $0.075^{\prime \prime}$ per pixel. Conventional MIR imaging was used in the observations from 5 June 2006, with standard data reduction, as described in Schödel et al. (2007), who used the same June 2006 data. During the selected observing runs in 2007, the so-called burst mode of VISIR was used (Doucet et al. 2006) to record individual short-exposure frames and the images were reconstructed with a speckle holography algorithm (see Liu \& Lohmann 1973; Bates et al. 1973; Petr et al. 1998)

In the standard imaging mode of VISIR, several tens of exposures (with detector integration times around $20 \mathrm{~ms}$ ) are averaged by the camera electronics before they are stored on disk. The burst mode suppresses the averaging and allows one to store the individual frames and thus to use VISIR as a speckle camera. Speckle image reconstruction methods can subsequently be applied to the burst mode data, which can lead to significant improvement of the image quality (see Doucet et al. 2006) because the deteriorating effects of atmospheric turbulence can be partially compensated by the reconstruction process. A prerequisite for this technique is the presence of a sufficiently bright ( $\sim 5 \mathrm{Jy})$ point source reference in the FOV. For example, a typical data reduction method would be to apply a simple shift-andadd (SSA) procedure (see, e.g., Christou 1991) to the individual frames, possibly combined with frame selection. The result is an

\footnotetext{
${ }^{3}$ Based on observations collected at the European Organisation for Astronomical Research in the Southern Hemisphere, Chile under programmes 071.B-0365, 073.A-0442, 073.B-0665, 076.B-0715, 077.B0552, 077.B-0028, 179.B-0261, 079.B-0084, 079.B-0929, 279.B-5022, 081.B-0648, and 082.B-0952.

${ }^{4}$ Observations in June 2006 and May 2007 were done by the first author, the other data were obtained from the ESO Science Archive.
} 

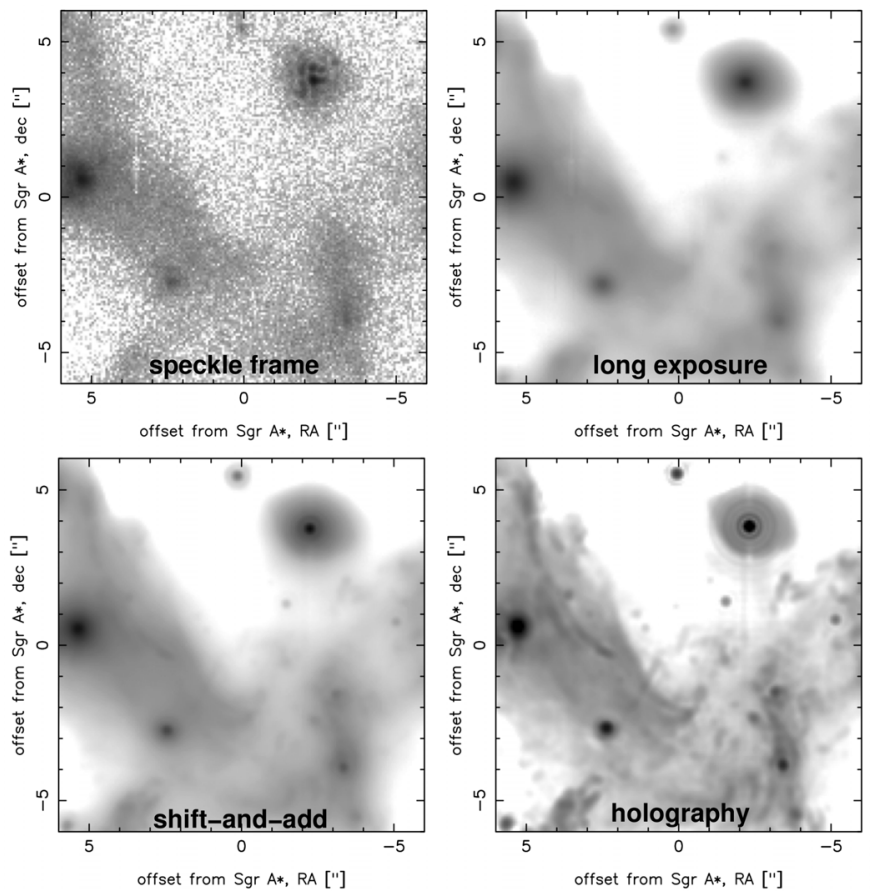

Fig. 1. Speckle imaging with VLT/VISIR at $8.59 \mu \mathrm{m}$; data from $21 / 22$ and 22/23 May 2007. Upper left: individual speckle frame. Upper right: long exposure image, i.e. straight average image with only a tiptilt correction applied, similar to standard imaging. Lower left: simple shift-and-add image. Lower right: image reconstructed with the speckle holography algorithm. the vertical line through IRS 3 visible in the lower right panel is an artifact of the detector.

increased Strehl ratio of the final image compared to a traditional long-exposure image.

The bright star IRS 3, located about 5" NW of Sgr A*, was used as point source reference for the speckle technique. The PSF of a point source in short exposure (burst mode) images in the MIR is usually dominated by a single speckle. However, the seeing during the May 2007 observations was so bad (visual seeing $\sim 2^{\prime \prime}$ according to the Paranal DIMM monitor) that IRS 3 appeared as a cloud of speckles in the majority of images (see upper left panel in Fig. 1). A simple shift-and-add (SSA) algorithm only takes advantage of the brightest speckle in the speckle cloud. The SSA algorithm can be improved through the selection of the best speckle frames, i.e. the ones with the highest $S / N$, which are short exposures of almost perfect image quality. However, the cost of such a lucky imaging technique is the loss of a large portion of the frames, typically 50\%-90\%, and thus a low efficiency. As an alternative, we chose to use the speckle holography technique for image reconstruction. Speckle holography uses the information in the entire instantaneous PSF (the speckle cloud) and therefore leads to images with high Strehl ratios without the need to discard any significant fraction of frames. A clear and concise description of the technique is presented in Petr et al. (1998). We used IRS 3 as PSF reference. The fact that IRS 3 is surrounded by a bow-shock of diffuse emission (see Fig. 1 or Viehmann et al. 2006) proved to be no impediment because the surface brightness of the diffuse flux is a factor $\sim 100$ fainter than the flux in the PSF of the point source and disappears within the noise in the individual speckle frames. The diffuse flux is therefore effectively suppressed by the noise threshold in our holography algorithm, that suppresses the noise in the determination of the instantaneous PSF.
The image reconstructed from the May 2007 burst mode data with the speckle holography algorithm is shown in the lower right panel of Fig. 1. For comparison, we show a single speckle frame in the upper left, the (tip-tilt corrected) long exposure image in the upper right, and the SSA image in the lower left panel. The image from the holography reconstruction is of extraordinary quality. This can be contrasted with the expectations from seeing-limited imaging: seeing in the visual was about $2^{\prime \prime}$ during the observations. The wavelength-dependence of seeing can be approximated with the Roddier formula, as $F W H M \propto \lambda^{-0.2}$, which predicts a $F W H M \sim 1^{\prime \prime}$ at $8.59 \mu \mathrm{m}$. However, the $F W H M$ of the reconstructed image is $0.25^{\prime \prime}$, the diffraction limit of the VLT at the observing wavelength. More than three diffraction rings are clearly visible around IRS 3 .

The VISIR burst mode observing template only provided chopping along the north-south (or east-west) direction; the chop throw was set to $\sim 15^{\prime \prime}$. We subtracted the flux-calibrated (see below) May 2007 image from the June 2006 image to estimate the possible systematic photometric uncertainty due to the non-ideal chopping. From a region approximately $5^{\prime \prime} \times 5^{\prime \prime}$ in size, centered on Sgr A*, we estimate an average uncertainty of the flux in each pixel of $\leq 10 \%$.

A deep image was created by combining all available burst mode frames from 2007 with the speckle holography algorithm for image reconstruction. A total of about 900000 frames with individual frame integration times of $0.016 \mathrm{~s}$ and $0.020 \mathrm{~s}$ were used, corresponding to a total integration time on target of roughly $4.5 \mathrm{~h}$. An issue with some of the April and July 2007 data, retrieved from the ESO archive, was the small chopping throw of just $10^{\prime \prime}$ in direction north-south. We checked whether this caused a significant flux bias in the area near Sgr A*, due to chopping into point-like or extended sources to the north or south. For this purpose we examined the difference between the images from just the May 2007 data, which should be free from significant errors due to chopping, and from all 2007 data. Within $\pm 1^{\prime \prime}$ from $\operatorname{Sgr} \mathrm{A}^{*}$ we found a negligible flux offset and a standard deviation between the pixel fluxes of $\leq 10 \%$. Therefore we can be confident that the flux calibration of all images reconstructed from the 2007 burst mode data is accurate to within at least $10 \%$.

The probably best observations (no chopping into obvious sources, large chopping throw, excellent seeing) are from 8 April 2007 ( 30000 frames) and are presented separately here because of their high quality.

The astrometric reference frame and absolute photometry were established using the imaging data from 4/5 June 2006. The reasons for this choice are the optimized chopping angle and throw of these data as well as the use of multiple dither positions. Additionally, both chopping and nodding were applied during these observations (only chopping in case of the burst mode observations). All these factors contribute to the suppression of systematic effects over a relatively large FOV. The StarFinder program package (Diolaiti et al. 2000) was used for measuring positions and fluxes of point sources as well as for obtaining a smooth estimate of the diffuse emission. Astrometric and photometric mean values and uncertainties were estimated by using two different PSFs, one determined from the star IRS 10EE, the only reasonably bright one in the MIR image that is not associated with evident diffuse emission, and one from the standard star HD 145897 (see below). The formal uncertainties calculated by StarFinder for each source were added in quadrature to the uncertainty estimated from the use of the two different PSFs.

The zero point for the $8.59 \mu \mathrm{m}$ PAH 1 filter was measured via observations of standard stars before, during, and at the 
end of the GC observations. The observations of the three standard stars HD 198048, HD 178345, and HD 145897 followed the ESO VISIR calibration plan. Zero points were determined using the visir_img_phot algorithm of the ESO Common Pipeline Library (CPL). The maximum deviation between the mean and the individual measurements was $3.2 \%$, indicating very stable atmospheric transmission during the entire observing time. The deviation from the zero point determined by Schödel et al. (2007), who used the same data but only one of the observed standard stars and a different algorithm for estimating the zero point, is also just $3 \%$. We adopt this value of $3 \%$ as conservative $1 \sigma$ uncertainty of the absolute photometric calibration of the PAH 1 image.

In a next step, the astrometric reference frame was established, i.e., pixel positions of stars were converted into offsets in arc-seconds from Sagittarius A*. To take possible optical distortion across the image into account, a polynomial with terms up to second order was used. The coefficients of the polynomial were computed via least-squares minimization between the pixel positions of 9 stars detected in the MIR image (IRS 15NE, IRS 7, IRS 3, IRS 29N, IRS 6E, IRS 12N, IRS 9, IRS 10EE, IRS 17) and known near-infrared positions and proper motions of the stars relative to $\mathrm{Sgr} \mathrm{A}^{*}$, as given in Schödel et al. (2009). The uncertainty of this transformation was determined with a Monte Carlo simulation. The astrometric positions of the reference stars were varied randomly in 100 tries within their $1 \sigma$ combined NIR and MIR positional uncertainties. The standard deviation of the resulting position for each star in the MIR frame was then adopted as the $1 \sigma$ uncertainty of its astrometric position and added quadratically to the uncertainty of its position in the field as measured by StarFinder.

A comparison of the positions of the maser stars (IRS 7, IRS 10EE, IRS 15NE, IRS 9, IRS 17) with the respective ones given in Schödel et al. (2009) showed a coincidence within the respective $1 \sigma$ uncertainties. The position of Sgr A* in the MIR image relative to prominent sources, like IRS $16 \mathrm{NW}$ or IRS 29, could be determined with an uncertainty of 0.04 pixel or about 3 mas. The absolute astrometric uncertainty of the stellar positions in the MIR image will be higher due to the absolute uncertainty of the NIR reference system used here. As illustrated in Fig. 5 of Schödel et al. (2009), the absolute astrometric uncertainty is thus on the order of $\sim 3$ milliarcseconds (mas) near Sgr A*, but as large as 50 mas at $\sim 15^{\prime \prime}$ distance from Sgr A*.

The flux density scale in all other images (from the 2007 data) was calibrated by using the fluxes of the sources IRS 2L, IRS 7, and IRS 13E as measured in the June 2006 image. The uncertainty of this procedure was estimated from the deviations of the fluxes of the three reference stars after calibration from their initially assumed fluxes. The uncertainty was found to be about $10 \%$. This uncertainty was added quadratically to the uncertainties of the PSF fitting. The FOV of the 2007 imaging data is smaller than the one of the 2006 data. Therefore, absolute astrometry was established via the astrometric positions of the sources IRS 7, IRS 3, IRS 29N, IRS 6E, IRS 21, and IRS 1W (Schödel et al. 2009). The position of Sgr A* in all images was determined with a $1 \sigma$ uncertainty of 0.04 pixels, or 3 mas. We detected no significant systematic differences between the photometric and astrometric calibration of the MIR images in this work and in our previous work (Schödel et al. 2007).

\subsection{Observations with $\mathrm{NaCo} / \mathrm{VLT}$ at $4.8 \mu \mathrm{m}$}

The central $\sim 15^{\prime \prime} \times 15^{\prime \prime}$ around Sgr A* were observed in $M^{\prime}$ with the near-infrared camera and adaptive optics system
Table 1. Flux density of $M^{\prime}$ counterpart of Sgr A*.

\begin{tabular}{|c|c|c|c|}
\hline UTC Date & UTC Time & $t_{\text {on source }}{ }^{a}$ & $f_{\mathrm{SgrA} *}{ }^{b}$ \\
\hline 2003 June 04 & $04: 21-07: 36$ & 130 & $1.6 \pm 0.2$ \\
\hline 2003 June 09 & $04: 29-07: 20$ & 115 & $1.2 \pm 0.2$ \\
\hline 2004 June 14 & $03: 30-05: 56$ & 58 & $1.8 \pm 0.3$ \\
\hline 2004 Aug. 11 & $00: 54-01: 15$ & 27 & $1.8 \pm 0.4$ \\
\hline 2004 Sep. 20 & $00: 06-01: 47$ & 55 & $1.6 \pm 0.2$ \\
\hline 2004 Sep. $20 / 21$ & $23: 29-00: 30$ & 95 & $1.2 \pm 0.2$ \\
\hline 2006 Mar. 28 & $07: 11-08: 49$ & 47 & $1.2 \pm 0.3$ \\
\hline 2006 Mar. 29 & $07: 06-10: 24$ & 122 & $1.8 \pm 0.2$ \\
\hline all $2003^{(c)}$ & & 245 & $1.4 \pm 0.2$ \\
\hline all $2004^{(c)}$ & & 235 & $1.6 \pm 0.2$ \\
\hline all $2006^{(c)}$ & & 169 & $1.6 \pm 0.2$ \\
\hline
\end{tabular}

Notes. ${ }^{(a)}$ Total integration time on source in seconds; ${ }^{(b)}$ flux density and $1 \sigma$ uncertainties in mJy, not corrected for extinction; ${ }^{(c)}$ measurement on average image from all 2003, 2004, or 2006 imaging data, respectively.

NAOS/CONICA (short: NaCo, see Lenzen et al. 2003; Rousset et al. 2003) at the ESO VLT at several epochs in 2003, 2004, and 2006 (Table 1). The data were obtained from the ESO science archive. Overall observing efficiency was low (due to chopping, dithering, read-out overheads, and possible other reasons unknown to the authors), so that the total on-source integration time of the $M^{\prime}$-band images was between half a minute and two minutes per observing epoch (Table 1). Astrometry was established via 13 sources in the FOV, using stellar positions and proper motions from Schödel et al. (2009). The resulting $1 \sigma$ uncertainty of the pixel position of $\mathrm{Sgr} \mathrm{A}^{*}$ in the $M^{\prime}$-images is $0.05-0.08$ pixels (1.2-2.2 mas) in both axes.

Photometry and astrometry of point sources in the $M^{\prime}$-image were performed with StarFinder. Absolute astrometry was established with the same sources as for the MIR observations. The absolute astrometric uncertainty is estimated to be better than 0.05" (Schödel et al. 2009). Since Sgr A* is clearly identifiable in $M^{\prime}$, very high astrometric precision is not a real issue at this wavelength (see Sect. 3.3 and Hornstein et al. 2007).

No measurement of the NACO $M^{\prime}$ zero point could be found on the ESO archive. Therefore, the stars IRS 16C and IRS 16NW were used to calibrate the zero point of the $M^{\prime}$ image. Their magnitudes are $L^{\prime}=8.20 \pm 0.15$ and $\mathrm{PAH} I=8.05 \pm 0.1$ for IRS 16C, and $L^{\prime}=8.43 \pm 0.15$ and PAH $I=8.67 \pm 0.1$ IRS 16NW. The $L^{\prime}$ magnitudes are from Schödel et al. (2010) and the PAH 1 magnitudes from photometry on the $20068.6 \mu \mathrm{m}$ data (see previous section). We assume that extinction stays approximately constant between the $L^{\prime}$-band and $8.6 \mu \mathrm{m}$ (see Lutz 1999; Nishiyama et al. 2009). It appears that both stars do not show any significant MIR excess and can be approximated as black bodies. We therefore assume the same magnitude in $M^{\prime}$ as in $L^{\prime}$ for both stars and use them to calibrate the $M^{\prime}$ image. The systematic $1 \sigma$ uncertainty of the $M^{\prime}$ calibration is $\sim 0.15$ mag and results from the combination of an estimated 0.1 mag uncertainty in extinction and $0.1 \mathrm{mag}$ uncertainty from the $L^{\prime}$ calibration (from the average of two sources). Hornstein et al. (2007) used an independent calibration procedure and obtained for IRS 16C and IRS 16NW $M_{\mathrm{S}}=8.08$ and $M_{\mathrm{S}}=8.41$, respectively. We use as flux density zero point $160 \mathrm{Jy}$ (Cohen et al. 1992), they used 163 Jy (Tokunaga \& Vacca 2005).

\subsection{Observations with $\mathrm{NaCo} / \mathrm{VLT}$ at $3.8 \mu \mathrm{m}$}

The central parsec around Sgr A* has frequently been observed with $\mathrm{NaCo} / \mathrm{VLT}$ since 2002. In order to constrain the emission of 
Table 2. Observations of the GC in the $L^{\prime}$-band with $\mathrm{NaCo}$, used in this work.

\begin{tabular}{lcccccc}
\hline \hline UTC Date & UTC Time & Good data $^{a}$ & $t_{\text {on source }}{ }^{b}$ & $f_{\text {mean,SgrA* }}{ }^{c}$ & $f_{\text {median,SgrA* }}{ }^{d}$ & $f_{\mathrm{S} 2}{ }^{e}$ \\
\hline 2006 Mar. 28 & $05: 60-10: 29$ & 0.74 & 850.5 & 0.64 & 0.82 & 1.47 \\
2006 May 29 & $04: 39-10: 51$ & 0.45 & 2400 & 1.21 & 0.97 & 1.86 \\
2006 May 30 & $06: 05-10: 24$ & 0.52 & 2400 & 1.72 & 1.62 & 1.96 \\
2006 May 31 & $08: 29-10: 37$ & 0.76 & 2490 & 1.65 & 1.65 & 2.07 \\
2006 Jun. 02 & $04: 47-07: 01$ & 0.53 & 1890 & 2.23 & 2.10 & 1.88 \\
2006 Jun. 04 & $05: 03-10: 22$ & 0.40 & 3360 & 1.86 & 1.85 & 2.10 \\
2006 Jun. 06 & $04: 49-10: 33$ & 0.39 & 3360 & 1.11 & 1.04 & 2.02 \\
2007 Apr. 01 & $05: 18-06: 43$ & 0.95 & 3420 & 0.92 & 0.90 & 1.85 \\
2007 Apr. 03 & $04: 57-06: 27$ & 1.00 & 3600 & 1.17 & 1.22 & 1.86 \\
2007 Apr. 04 & $04: 50-06: 10$ & 0.93 & 3000 & 3.14 & 3.08 & 1.66 \\
2007 Apr. 05 & $08: 01-10: 35$ & 0.88 & 4890 & 1.51 & 1.39 & 2.08 \\
2007 Apr. 06 & $05: 19-06: 55$ & 0.89 & 3210 & 0.72 & 0.74 & 1.68 \\
2007 May 23 & $04: 39-10: 36$ & 0.26 & 3060 & 1.82 & 1.57 & 2.16 \\
2007 Jul. 20 & $01: 35-02: 11$ & 0.94 & 1350 & 1.25 & 1.11 & 2.06 \\
2007 Jul. 22 & 23:08-04:51 & 0.88 & 7590 & 1.30 & 1.16 & 1.97 \\
2008 May 26 & $05: 43-10: 38$ & 0.60 & 3720 & 3.93 & 3.97 & 1.88 \\
2008 May 30 & $06: 41-10: 41$ & 0.54 & 1470 & 1.27 & 1.18 & 1.99 \\
2009 Apr. 01 & $06: 11-09: 01$ & 0.95 & 5700 & 1.69 & 1.54 & 1.63 \\
2009 Apr. 05 & $07: 02-10: 35$ & 0.62 & 3060 & 0.91 & 0.90 & 1.76 \\
\hline
\end{tabular}

Notes. The detector integration time (DIT) was $0.2 \mathrm{~s}$ in all cases, except 28 Mar. 2006, when it was $0.175 \mathrm{~s}$; ${ }^{(a)}$ fraction of data used; rest rejected because of low quality; ${ }^{(b)}$ total integration time on source in seconds, excluding rejected data; ${ }^{(c)}$ measured flux density of Sgr A* on mean images, in mJy, not corrected for extinction; ${ }^{(d)}$ measured flux density of Sgr A* on median images, in mJy, not corrected for extinction; ${ }^{\left({ }^{*}\right)}$ measured flux density of $\mathrm{S} 2$ on mean images, in mJy, not corrected for extinction.

Sgr A* in the $L^{\prime}$-band $(3.8 \mu \mathrm{m})$, we downloaded $L^{\prime}$-imaging data from the years 2006-2009. The principal reason to choose this time window is that $\mathrm{S} 2 / \mathrm{S} 0-2$, the brightest star near Sgr A*, was relatively far from the black hole during this period. The small proper motion of S2 near the apocenter of its orbit also ensures that the star will hardly appear blurred on images created from averaging the data from different epochs. No selection criterion was applied to the data other than imaging quality (atmospheric conditions, AO performance). Particularly important is that the flux state of $\operatorname{Sgr~A}^{*}$ was not used as a criterion to include or exclude a particular data set.

Data reduction was standard (sky subtraction, bad pixel interpolation, flat-fielding). Epochs of low data quality, e.g., because of strongly variable transmission, extreme turbulence and consequentially insufficient $\mathrm{AO}$ correction, or strong readout patterns on the detector, were discarded. In the data from the remaining epochs, frames with insufficient AO performance (sometimes the AO loop opens), insufficient removal of skybackground (due to rapidly variable background in the $L^{\prime}$-band) or otherwise corrupted frames were de-selected before creating final mosaics. The percentage of discarded frames ranges between $0 \%-70 \%$, depending on the observing epoch. For AO observations of the GC with $\mathrm{NaCo} / \mathrm{VLT}$ the $L^{\prime}$-band is frequently used as fall-back option when atmospheric conditions are bad, particularly when seeing is bad and fast. It is for this reason that the number of discarded frames is very high for several epochs. The selected observing epochs, along with the total exposure time for each one (excluding de-selected frames) are listed in Table 2.

Photometry and astrometry of point sources in the $L^{\prime}$-image were performed with StarFinder. Absolute astrometry was established with the same sources as for the MIR observations. The absolute astrometric uncertainty is estimated to be better than 0.05" (Schödel et al. 2009). Since Sgr A* is clearly identifiable in $L^{\prime}$, very high precision in astrometry is not a real issue at this wavelength. Photometric calibration is based on the magnitudes of IRS 16C and IRS 16NW provided by Schödel et al. (2010).

\section{The mean emission from Sgr $A^{*}$ in the infrared}

\subsection{The immediate environment of $\operatorname{Sgr} A^{*}$ at $8.6 \mu \mathrm{m}$}

Figure 2 shows the surroundings within $\pm 2^{\prime \prime}$ of $\mathrm{Sgr}^{*}$ on images reconstructed from data obtained on 5 June 2006, 22/23 May 2007, 8 April 2007, and all 2007. The structures seen on all images are very similar. The images also resemble closely the ones shown in Figs. 1 and 2 of Schödel et al. (2007), who used the same June 2006 observations as in this paper and applied Lucy-Richardson deconvolution. We attribute any obvious differences to the fact that the images have different signal-to-noise ratios, with the highest $S / N$ reached in the image reconstructed from the entire 2007 burst mode data set. Also, the June 2006 image from direct imaging has a lower Strehl ratio than the images from 2007 that are based on burst mode observations and reconstructed via the speckle holography algorithm. The excellent quality of the new images makes the application of deconvolution unnecessary, thus avoiding any related potential complications.

As can be seen, Sgr A* is not isolated, but located near the middle of a ridge-like structure, which we term the $S g r *^{*}$-Ridge. It is marked by a dashed oval in the upper right panel of Fig. 2. There is no recognizable point-source coincident with its position, but there may be a point source that is confused with diffuse emission. In order to estimate an upper limit to the mean flux density of $\mathrm{Sgr}^{*}$, we need a reasonable estimate of the probable diffuse background flux density at its location.

\subsection{An upper limit on the mean emission from $\operatorname{Sgr} A^{*}$ at $8.6 \mu \mathrm{m}$}

The upper right, and lower left and right panels of Fig. 3 show the image reconstructed from all 2007 data with a point source (convolved with the corresponding PSF) of 5, 10, and $15 \mathrm{mJy}$ subtracted from the position of Sgr A*. At flux densities $\gtrsim 10 \mathrm{mJy}$ a depression becomes apparent at the position of Sgr A*. Therefore, we judge that the flux density of Sgr A* must 

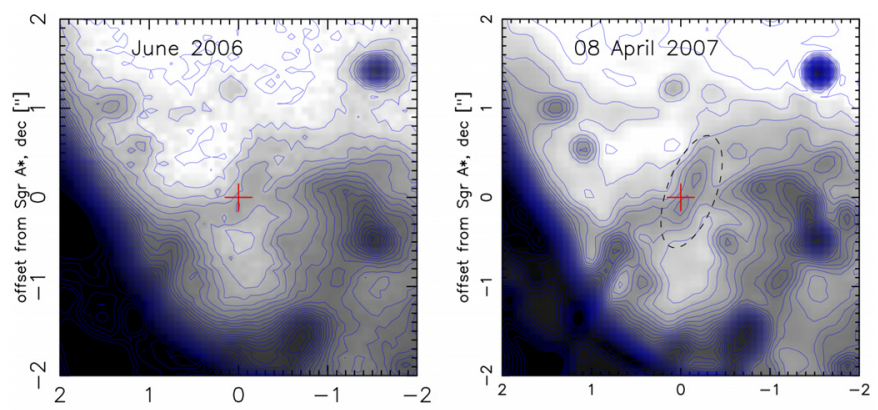

offset from Sgr A*, RA ["]
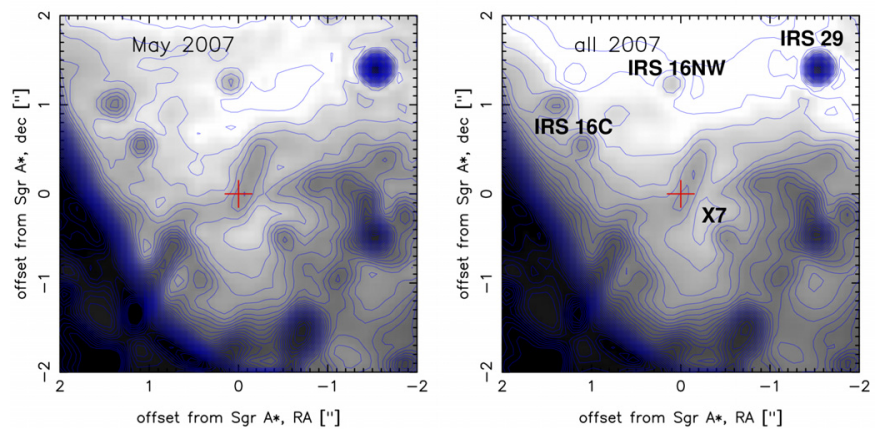

Fig. 2. The surroundings of Sgr A* at $8.6 \mu \mathrm{m}$. Upper left: image from long-exposure observations on 5 June 2006. Upper right: image reconstructed with the speckle holography algorithm from burst mode observations on 8 April 2007. Lower left: image reconstructed with the speckle holography algorithm from burst mode observations on 22 and 23 May 2007. Lower right: image reconstructed with the speckle holography algorithm from all burst mode observations in 2007. North is up and East is to the left. Contour lines are plotted in steps of $0.5 \mathrm{mJy}$ from 0.5 to $20 \mathrm{mJy}$ per pixel (one pixel corresponds to $0.075^{\prime \prime} \times 0.075^{\prime \prime}$ ). $\mathrm{Sgr}^{*}$ is marked with a red cross of $0.3^{\prime \prime}$ line segments. The stellar sources IRS16 NW, IRS16 C, and IRS29, as well as the cometary shaped source X7 (Mužić et al. 2010) are labeled in the lower right

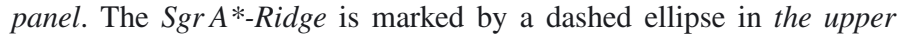
right panel.

be $\lesssim 10 \mathrm{mJy}$. Another way of estimating the emission from a putative Sgr A* counterpart is by estimating the background emission at its location and subtracting it from the images.

The background emission at the position of $\mathrm{Sgr} \mathrm{A}^{*}$ can be estimated by masking the pixels in a circular area of a few pixels radius, centered on $\mathrm{Sgr} \mathrm{A}^{*}$, and subsequently interpolating the flux in the masked pixels with the StarFinder (Diolaiti et al. $2000)$ routine REPLACE_PIX. This routine replaces bad pixels with a median of the pixel values in a suitably large box around the corresponding location. A minimum of three "good" pixels is used to compute the median value at a given location. For details, see the publicly available source code of StarFinder. For the 2007 images, a masking radius of $3(2,4)$ pixels contains about $78 \%(62 \%, 80 \%)$ of the flux on from a putative point source at the position of $\mathrm{Sgr} \mathrm{A}^{*}$. For a radius 3 pixels mask any pixel outside this radius would be contaminated by $<5 \%$ of the peak pixel flux of such a point source. Therefore, this procedure should effectively remove any influence from an undiscovered point source at the position of Sgr A*. An example of the interpolated background is shown in the upper left panel of Fig. 3, using the image reconstructed from all 2007 data.

The estimated background can subsequently be subtracted from an image and the remaining flux at the position of Sgr A* can be measured and corrected for the used aperture (a stronger correction was used for the 2006 image with its lower Strehl ratio). Due to the emission from the SgrA*-Ridge, the estimated
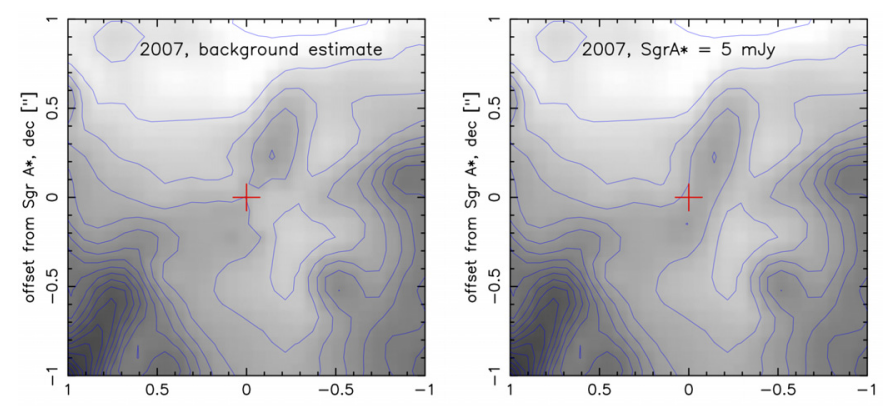

offset from Sgr A*, RA ["]
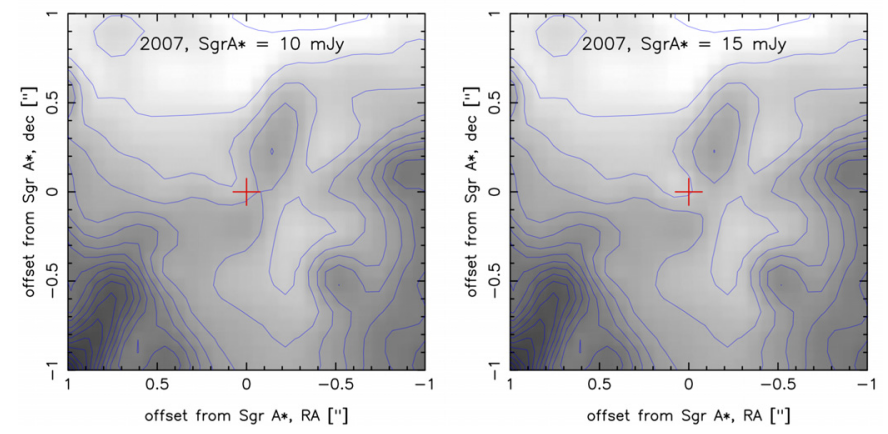

Fig. 3. Zoom onto $\mathrm{Sgr}^{*}$ at $8.6 \mu \mathrm{m}$ in the image reconstructed with the speckle holography algorithm from all burst mode observations in 2007. North is up and East is to the left. Contour lines are identical to the ones in Fig. 2. Sgr A* is marked with a cross of $0.075^{\prime \prime}$ line segments. Upper left panel: estimate of the diffuse background emission at the position of Sgr A*. A point source of 5, 10, and $15 \mathrm{mJy}$ was subtracted from the original reconstructed image at the position of $\operatorname{Sgr} \mathrm{A}^{*}$ with the results shown in the upper right, lower left, and lower right panels, respectively.

Table 3. Remnant $8.6 \mu \mathrm{m}$ flux densities at the position of Sgr A* after background subtraction.

\begin{tabular}{lcc}
\hline \hline $\begin{array}{l}\text { Date } \\
(\mathrm{UT})\end{array}$ & $\begin{array}{c}f \\
(\mathrm{mJy})\end{array}$ & $\begin{array}{c}d f \\
(\mathrm{mJy})\end{array}$ \\
\hline 2006 May 5 & 5.9 & 1.2 \\
2007 Apr. 1 & 2.7 & 1.3 \\
2007 Apr. 3 & 4.5 & 1.7 \\
2007 Apr. 5 & 2.4 & 1.1 \\
2007 Apr. 6 & 3.6 & 2.1 \\
2007 Apr. 8 & 7.9 & 4.2 \\
2007 May 22 & 7.6 & 3.3 \\
2007 May 23 & 8.3 & 2.5 \\
2007 Jul. 20 & 10.1 & 3.9 \\
2007 Jul. 21 & 10.1 & 4.2 \\
2007 Jul. 23 & 8.8 & 3.6 \\
2007 Jul. 24 & 8.3 & 3.4 \\
\hline
\end{tabular}

background flux will depend on the masking radius. As can be seen in the upper left panel of Fig. 3, a radius 3 pixels mask will effectively remove the ridge emission at the position of Sgr A*. A 2 pixels masking radius will include more of the ridge emission into the background flux at the position of $\operatorname{Sgr} \mathrm{A}^{*}$, while a 4 pixels masking radius will probably under-estimate the background emission at the position of $\operatorname{Sgr} \mathrm{A}^{*}$. We use these three different masking radii to measure the remnant flux and estimate its uncertainty.

This procedure was applied to the images from all epochs. The measured remnant fluxes at the position of Sgr A* as well as the corresponding uncertainties are listed in Table 3 . The mean of all 12 independent measurements is $6.7 \pm 0.7 \mathrm{mJy}$, while the 
weighted mean is $4.5 \pm 0.5 \mathrm{mJy}$. However, the measurements from 1, 3, 5, and 6 April 2007 appear to be systematically low. When inspecting those images, very similar patterns of negative emission can be seen in large parts of the images, also close to $\mathrm{Sgr} \mathrm{A}^{*}$. A further examination of the corresponding imaging data reveals that the chopping angle was chosen along a N-S direction with an amplitude of just $8^{\prime \prime}$. The effect is that bright sources, like IRS 7, IRS 4, IRS 21, and much extended emission from the northern and southern parts of the mini-spiral are subtracted from the source images. It is possible that the Sgr A* region and/or the region of the calibrator sources are affected by these systematic negativities. We therefore discard these measurements, which is a conservative step because it will correct the estimated mean flux upwards. After exclusion of the problematic April 2007 data sets, we obtain a mean from 8 independent measurements of $8.4 \pm 0.5 \mathrm{mJy}$, where the weighted mean is $7.1 \pm 0.9 \mathrm{mJy}$.

Schödel et al. (2007) reported $8 \pm 5 \mathrm{mJy}$ as the flux density of any putative point source at the position of Sgr A*. At first glance, there seems to be hardly any difference in the results between this work and the earlier one. However mid-infrared observations of the Galactic center are non-trivial because of the involved systematic uncertainties (see problem of systematic errors related to non-optimal chopping described above). Furthermore, there is no straightforward way to measure the upper limit of the emission from a putative point-like source at the position of $\operatorname{Sgr} \mathrm{A}^{*}$. The method applied here is different and independent from the one applied in Schödel et al. (2007), who subtracted the flux-scaled diffuse emission of a point-source subtracted $L^{\prime}$-band image from the MIR image. The quality and quantity of analyzed data is much higher here. The zero point is established with greater reliability via the use of three instead of just one standard stars. Finally, the use of many different epochs with different observing setups (particularly the chopping angle) will minimize the influence of systematic errors. The fact that the result here is similar to that in our previous work gives us great confidence in the estimated upper limit, while the uncertainty has been reduced significantly.

The measured remnant flux can be regarded as an estimate of the upper limit to any potential point-source at the position of Sgr A*. The deep images produced in this work show no indication of any point source at $8.6 \mu \mathrm{m}$ at the position of $\operatorname{Sgr} \mathrm{A}^{*}$. It is possible that the dust emission at the position of $\mathrm{Sgr} \mathrm{A}^{*}$ reaches levels similar to those measured in the parts of the $\operatorname{Sgr} A^{*}$-Ridge immediately NW and SE of Sgr A*. In this case, the background estimates obtained with a 2 pixel masking radius would be most appropriate, resulting in an estimated remnant flux of just $3 \mathrm{mJy}$. Since two of the three masking apertures used here are larger, we are confident that our measurements are conservative. We may even overestimate the remnant flux at the position of Sgr A* by factors $2-3$.

Hence, from the weighted mean, we derive a $3 \sigma$ upper limit to the long-term average of the flux density of Sgr A* of $9.8 \mathrm{mJy}$ ( $9.9 \mathrm{mJy}$ for the unweighted mean).

Unfortunately, $\lambda=8.6 \mu \mathrm{m}$ is located right on the blue edge of the $10 \mu \mathrm{m}$ silicate absorption feature (see, e.g., Moneti et al. 2001; Lutz et al. 1996), giving rise to relatively high extinction. Here, we use $A_{8.6} \approx 2.0 \pm 0.3$, from the recent measurements by Fritz et al. (2011). The extinction corrected upper limit on the $8.6 \mu \mathrm{m}$ emission from $\mathrm{Sgr} \mathrm{A}^{*}$ is thus $45 \pm 13 \mathrm{mJy}$, using the weighted mean. We note that the extinction assumed here is higher than what was used by Schödel et al. (2007). Their upper limit, corrected for the extinction used here, is $50 \pm 33 \mathrm{mJy}$. In the
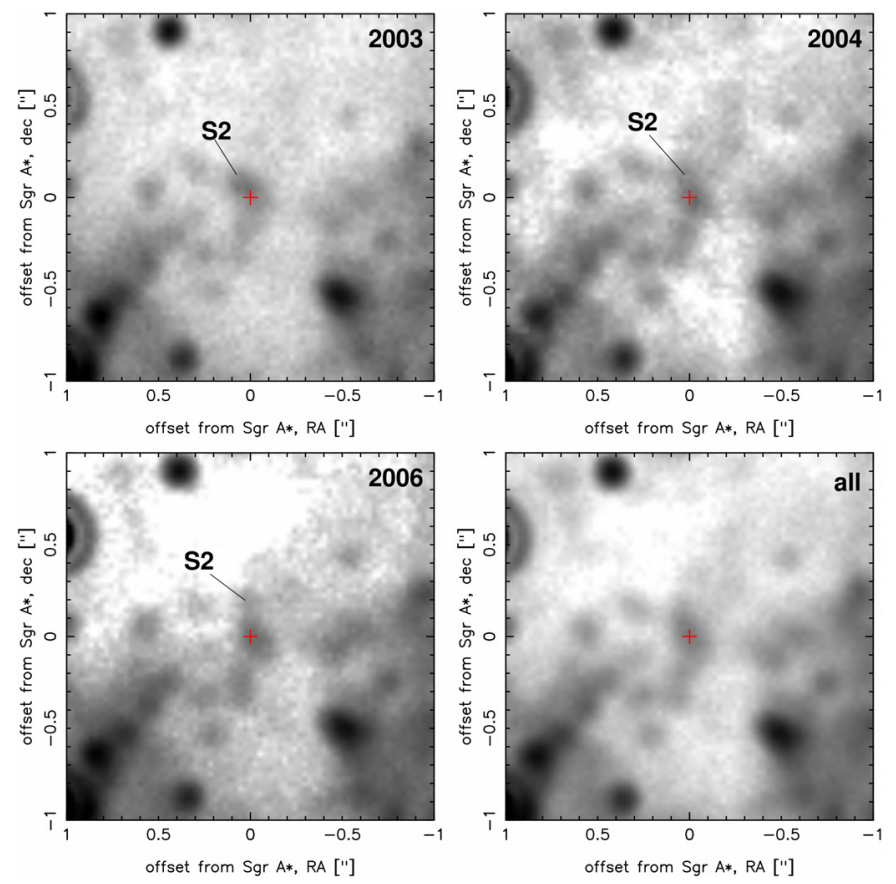

Fig. 4. The surroundings of $\mathrm{Sgr} \mathrm{A}^{*}$ seen with $\mathrm{NaCo}$ in the $M^{\prime}$-band. Upper left: average image from 2003 data. Upper right: average image from 2004 data. Lower left: average image from 2006 data. Lower right: average image from 2003-2006 data. Sgr A* is marked with a red cross. The fast-moving star S2 is marked in the 2003, 2004, and 2006 images. The gray scale is logarithmic.

following, we will use a de-reddened $3 \sigma$ upper limit of $84 \mathrm{mJy}$, which includes the uncertainty of the extinction correction.

\subsection{The mean emission from $\operatorname{Sgr} A^{*}$ at $4.8 \mu \mathrm{m}$}

The average images from the 2003, 2004, and $2006 \mathrm{NaCo}$ $M^{\prime}$-band imaging data are shown in Fig. 4 together with an average image based on the data from all epochs. As can be seen, a source can clearly be detected at the location of Sgr A* in the $M^{\prime}$-band on all images. The source near Sgr A* appears to be elongated toward the SE at all epochs. This is likely due to emission from a compact dust blob located about 0.094" to the SE of Sgr A*. This extended source was reported by various authors and has an approximate $L^{\prime}$-magnitude of 12.8-13.7 (e.g., Clénet et al. 2004; Ghez et al. 2005; Eckart et al. 2006a; Hornstein et al. 2007). According to Ghez et al. (2005), the dust blob can be modeled as a two-dimensional Gaussian with a major axis of 120 mas. This is less than the diffraction-limited resolution of $\mathrm{NaCo}$ at $4.8 \mu \mathrm{m}$ ( $\sim 125$ mas $)$.

Because of the closeness of the dust blob to Sgr A*, the two sources could not be separated by StarFinder. In order to estimate the flux of Sgr A*, we therefore proceeded in the following way. Using the PSF extracted from the $M^{\prime}$-images, two point sources were subtracted from the images: one at the position of Sgr A* and one at the position of the dust blob, 0.094" SE of Sgr A* (Ghez et al. 2005). The flux of the sources was varied until, after subtraction, the emission at the corresponding positions appeared flat and equaled approximately the surrounding background (see Fig. 5). The best fit and the uncertainties were estimated by eye. We could not detect any significant extended residuals after subtracting Sgr A* plus the dust blob from the images. We therefore estimate that the assumption of a point-like source for the dust blob is a good approximation. 

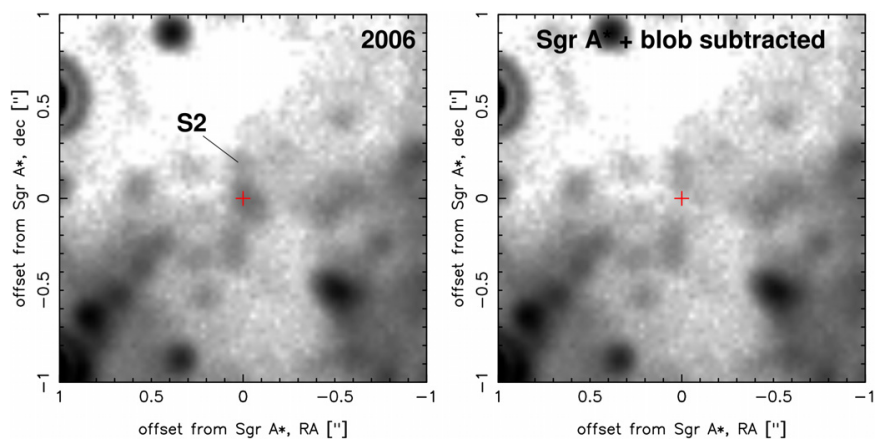

Fig. 5. Left: average $M^{\prime}$-image from 2006 data. Right: the same image, after subtraction of two point sources, one at the position of Sgr A* and one $\sim 0.094^{\prime \prime}$ to the SE of Sgr A*. The position of Sgr A* is indicated by a red cross.

In this way the flux density of the dust blob is estimated to be $1.2 \pm 0.3 \mathrm{mJy}$. This agrees well with the $0.5-1.2 \mathrm{mJy}$ that were reported for its emission at $L^{\prime}$. The flux density of the point source at the position of $\mathrm{Sgr} \mathrm{A}^{*}$ varies between 1.2 and $1.8 \mathrm{mJy}$. The individual measurements for Sgr A* are listed in Table 1. If no source at the position of the dust blob were subtracted from the images, the measured flux densities of Sgr A* would be about $0.3-0.4 \mathrm{mJy}$ higher. From this low contamination and from some experimenting with variable positions and flux densities for the dust blob, we estimate that any errors on the assumed position and flux of the dust blob, including its approximation as a point source, will result in systematic errors not larger than about $0.1 \mathrm{mJy}$ on the estimated flux density of $\operatorname{Sgr} \mathrm{A}^{*}$.

Within the uncertainties there is no significant variability of the putative $M^{\prime}$-counterpart of $\mathrm{Sgr}$ A* between the observing epochs. This does not exclude significant variability on timescales of several 10 to $100 \mathrm{~min}$ as is reported for the $L^{\prime}$-counterpart of Sgr A* (e.g., Ghez et al. 2005; Eckart et al. 2008b; Dodds-Eden et al. 2009) and has also been found in the $M$-band (Hornstein et al. 2007). Here, we did not try to examine the variability of $\mathrm{Sgr} \mathrm{A}^{*}$ at $M^{\prime}$ on short timescales. Such observations appear to be extremely difficult with NaCo. Chopping was used for all observations. Due to the small chopping throw in the available data and the crowded GC region we found that any reliable $M^{\prime}$-image must be composed of data at different dither positions. Chopping is not offered any more at the UT of the VLT where NaCo is mounted.

A final question is whether the $M^{\prime}$ emission from $\mathrm{Sgr}$ A* is contaminated by emission from stars in the extremely dense cluster of stars around the black hole. The stars in the so-called S-cluster are mainly B-type main-sequence stars (Gillessen et al. 2009). Sabha et al. (2010) report that unresolved stellar sources do not contribute more than a reddened $K$ s-band flux density of $0.15 \mathrm{mJy}$ at the position of Sgr A*. This corresponds to a mere $0.16 \mathrm{mJy}$ in $M^{\prime}$ (see below for the extinction in $M^{\prime}$ ). As concerns resolved sources, we can safely assume that no star brighter than $K \mathrm{~s} \approx 15.5$ (about $0.3 \mathrm{mJy}$ ) was confused with $\mathrm{Sgr} \mathrm{A} *$ during the $M^{\prime}$ observations (inferred from stellar orbits, see, e.g., Gillessen et al. 2009). We thus assign a conservative $0.3 \mathrm{mJy}$ as possible systematic $1 \sigma$ uncertainty due to contamination by stellar flux.

The time-weighted mean of all our measurements of the putative $M^{\prime}$ counterpart of $\operatorname{Sgr} \mathrm{A}^{*}$ is $1.51 \mathrm{mJy}$. The corresponding (time-weighted) uncertainty is $0.10 \mathrm{mJy}$. The systematic $1 \sigma$ uncertainty due to possible contamination by stellar flux or from the dust blob is $0.3 \mathrm{mJy}$, as mentioned above. For the following analysis, we combine the statistical and systematic uncertainties in quadrature and obtain $0.32 \mathrm{mJy}$. Estimating
$A_{M^{\prime}} / A_{K \mathrm{~s}}=0.4 \pm 0.05$ and its uncertainty from Nishiyama et al. (2009) and using $A_{K \mathrm{~s}}=2.46 \pm 0.03$ toward Sgr A* from Schödel et al. (2010) we get $A_{M^{\prime}}=1.0 \pm 0.3$. We thus obtain an extinction corrected flux density of $3.8 \pm 1.3 \mathrm{mJy}$ for the $M^{\prime}$ counterpart of $\operatorname{Sgr} A^{*}$, where the uncertainty of the extinction correction was taken into account. The $M^{\prime}$-flux density inferred in this work is similar to the one measured by Clénet et al. (2004), although they calibrate the zero point and extinction correction in a different way than in this work. Clénet et al. (2004) do not discuss the influence of the nearby dust blob, which was only discovered in later work and is probably the cause of the astrometric offset of the Sgr A* source discussed by Clénet et al. (2004). The similar photometric results confirm the robustness of the photometry of $\mathrm{Sgr} \mathrm{A}^{*}$ in $M^{\prime}$ and that the systematic uncertainty due to the nearby dust blob is not significant. Finally, as reported by Dodds-Eden et al. (2011) for the $K$ s-band and shown below for the $L^{\prime}$-band, Sgr A* spends only a small fraction of the time in high flux states. Therefore, sampling 8 random epochs ( 9 when including the results of Hornstein et al. 2007, see below), will probably provide a reasonable estimate of the mean emission of Sgr A* in the $M^{\prime}$-band. We note that the flux densities from the 8 VLT epochs agree within $\sim 1 \sigma$ of the individual uncertainties.

Hornstein et al. (2007) reported an extinction corrected flux density of $7.3 \pm 1.7 \mathrm{mJy}$. This difference can probably not be explained by errors in calibration because our photometry agrees well with the one of Hornstein et al. (2007, see also Sect.2.2). Some of the difference may be attributed to use of different extinction laws. Applying the extinction law used by Hornstein et al. (2007) to our measurements, we obtain $5.8 \pm 2.0 \mathrm{mJy}$ for the extinction corrected flux density from Sgr A*. Hence, the de-reddened flux densities agree within their $1 \sigma$ uncertainties. In this paper we report the mean flux of Sgr A* averaged over a large data set covering several epochs, while Hornstein et al. (2007) report the mean emission during just a single epoch. Sgr A* was probably more active during their observations; in fact, the light curve presented in Hornstein et al. (2007) shows clear signs of ongoing activity. Since the total exposure time of their data is much longer than the one of the VLT data, including their value into our data set would mean that it completely dominates the statistics. We therefore refrain from mixing the two data sets because we do not know whether the event observed by Hornstein et al. (2007) was typical or exceptional. Nevertheless, if we include their data, this changes the extinction-corrected mean $M^{\prime}$ flux density of $\mathrm{Sgr} \mathrm{A}^{*}$ to $4.6 \pm 1.5 \mathrm{mJy}$, i.e. the difference is not significant. In any case, the independent observations by Hornstein et al. (2007) support the notion that the mean flux of $\mathrm{SgrA}^{*}$ in the $M^{\prime}$-band measured in the $\mathrm{NaCo} / \mathrm{VLT}$ data is rather typical.

\subsection{The mean emission from $\operatorname{Sgr} A^{*}$ at $3.8 \mu \mathrm{m}$}

Photometry on the $L^{\prime}$-images from all available epochs was done with StarFinder. The PSF was extracted by using 14 reference stars within about $8^{\prime \prime}$ of Sgr A*. The correlation threshold was set to 0.7 , and the back_box parameter was set to 2 pixels.

Two iterations were run on each image with a $3 \sigma$ noise threshold. Photometry was calibrated by using the two stars IRS $16 C$ and IRS $16 N W^{5}\left(L^{\prime}=8.20,8.43\right.$, Schödel et al. 2010).

The fourth and the sixth columns in Table 2 list the measured fluxes of the star S2/S0-2 and of Sgr A*. We believe

5 This star is classified as variable by Rafelski et al. (2007), but we consider the small reported variability negligible for the purpose here. Ott et al. (1999) did not report IRS 16NW as variable. 

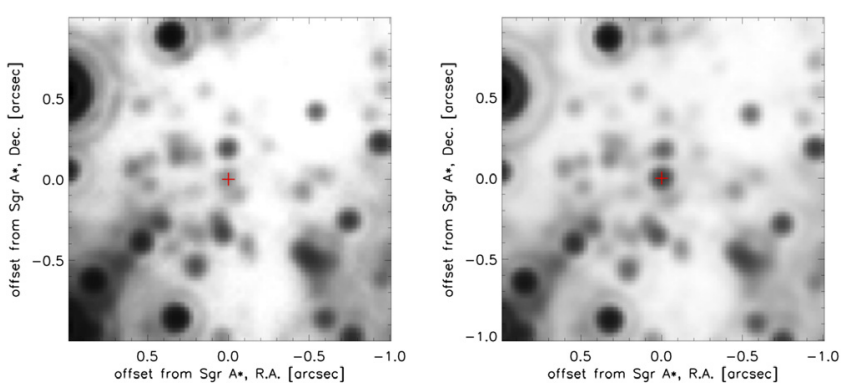

Fig. 6. Left: $L^{\prime}$-image from 1 April 2007, one of the epochs with the faintest mean state of Sgr A*. Right: $L^{\prime}$-image from 26 May 2008, one of the epochs with the brightest mean state of Sgr A*. The position of Sgr A* is indicated by a red cross.

that contamination of the measured fluxes of $\mathrm{Sgr} \mathrm{A}^{*}$ and $\mathrm{S} 2$ by point sources or by unresolved stars is negligible because of the high Strehl ratio (estimated at $\gtrsim 70 \%$ ) of the $L^{\prime}$-images and the fitting of a finely sampled background emission. The unweighted means and the corresponding uncertainties of the flux densities are $f_{\mathrm{S} 2}=1.89 \pm 0.04 \mathrm{mJy}$ (corresponding to $L^{\prime}=12.79 \pm 0.02$ ) and $f_{\mathrm{SgrA} *}=1.58 \pm 0.19 \mathrm{mJy}$ (corresponding to $\left.L^{\prime}=12.99 \pm 0.13\right)$. The exposure-time-weighted means and corresponding uncertainties are $f_{\mathrm{S} 2}=1.90 \pm 0.02 \mathrm{mJy}$ (corresponding to $L^{\prime}=12.79 \pm 0.02$ ) and $f_{\mathrm{SgrA} *}=1.62 \pm 0.20 \mathrm{mJy}$ (corresponding to $L^{\prime}=12.96 \pm 0.13$ ). No variability of the star $\mathrm{S} 2 / \mathrm{S} 0-2$ has been reported so far. Its measured mean flux density is in excellent agreement with previously published results (e.g., $L_{\mathrm{S} 0-2}^{\prime}=12.78 \pm 0.03$ Ghez et al. 2005). The dispersion of measured flux densities of $\mathrm{SgrA}^{*}$ is greater by a factor of almost 20 than that of S2/S0-2, which clearly indicates the variability of this source. In the following, we will use the uncertainty of the mean emission of $\operatorname{Sgr~A}^{*}$, which is $0.20 \mathrm{mJy}$, and not the standard deviations calculated from the individual measurements. $L^{\prime}$-images of the close surroundings of $\mathrm{Sgr} \mathrm{A}^{*}$ are shown for two epochs in Fig. 6.

After applying an extinction correction of $A_{L^{\prime}}=1.23 \pm 0.08$ (Schödel et al. 2010), we obtain a de-reddened mean flux density of $5.0 \pm 0.6 \mathrm{mJy}$ for $\mathrm{Sgr} \mathrm{A}^{*}$ (uncertainty of the extinction correction was included in the uncertainty of the flux density).

In order to test whether the mean flux density is sensitive to rare events, like exceptionally bright and long flares (e.g., Dodds-Eden et al. 2009; Kunneriath et al. 2010), the flux density of Sgr A* was also measured on the median images of each epoch. As can be seen in Col. 5 of Table 2, the values are generally slightly lower, but hardly different from the values measured on the mean images.

Finally, we created subsets of the data from each epoch in order to construct 74 independent images from the existing data, of about $800 \mathrm{~s}$ exposure time each. The $800 \mathrm{~s}$ exposure time offers a good compromise between a relatively high time resolution, on the one hand, and the need to average a sufficient number of frames in order to create clean images, on the other hand. This is particularly important in the case of $L^{\prime}$-imaging because of the high thermal background and the difficulty of extracting accurately the instantaneous sky emission from a small number of dithered frames on an extended and bright target.

The 800 s-images were inspected by eye and analyzed with StarFinder. Sgr A* is detected in each image. The measured flux distributions of Sgr A* and of S2 are shown in Fig. 7. The histogram of the flux densities of the constant comparison star S2

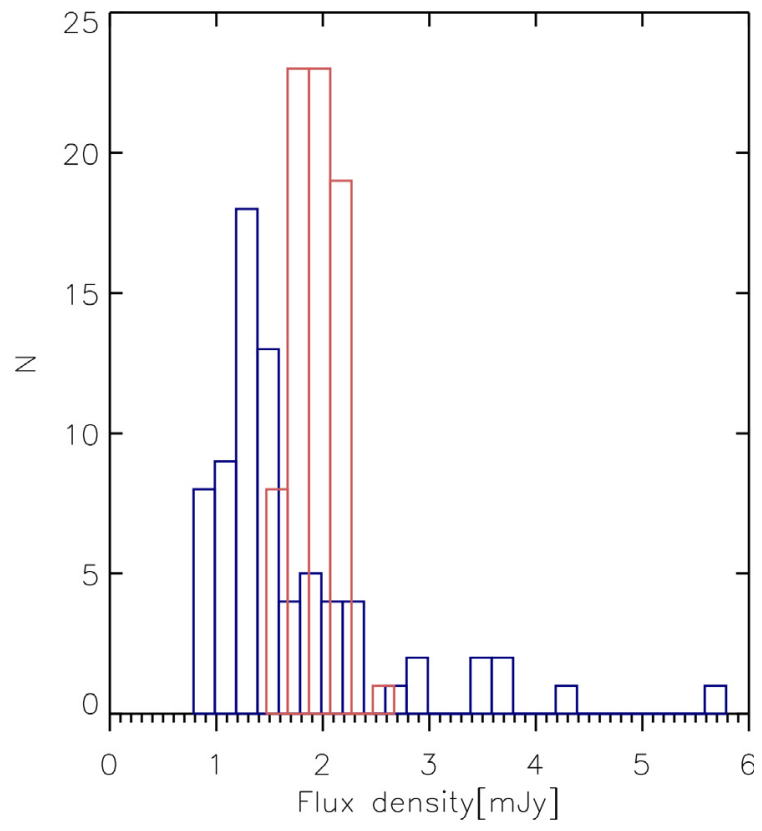

Fig. 7. Blue: histogram of measured $L^{\prime}$-flux densities (not corrected for extinction) of Sgr A* in $800 \mathrm{~s}$ exposures. Red: equivalent histogram for the constant star S2.

is close to Gaussian and provides an estimate of the photometric uncertainty. The histogram of $\mathrm{Sgr} \mathrm{A} *$ is significantly broader, with a tail toward high flux densities, similar to what has been found in a detailed analysis of the variability of Sgr A* in the $K$ s-band (Dodds-Eden et al. 2011). It is beyond the aims of this paper to discuss the exact time-variable behavior of Sgr A* in the $L^{\prime}$-band. Here, it is only important that $\mathrm{Sgr} \mathrm{A}^{*}$ is always detectable on images with integration times on the order of $13 \mathrm{~min}$. It appears to spend most of the time in a low state between about 1-2 mJy (not corrected for extinction), and shows only occasional excursions toward high flux values, potentially reflecting overlapping quiescent and flaring states (see Dodds-Eden et al. 2011). For example, the $3.8 \mu \mathrm{m}$ flux density of Sgr A* exceeds 2.0 (2.5) mJy (not corrected for extinction) in only $23 \%$ $(12 \%)$ of the 800 s-images. This underlines that it is meaningful to speak of a mean flux density of Sgr A* in the $L^{\prime}$-band.

It appears that, in the $L$-band, Sgr A* is continually variable on levels between 1 and several $10 \mathrm{mJy}$ (all flux densities extinction corrected, e.g., Ghez et al. 2005; Eckart et al. 2008b; Dodds-Eden et al. 2009). Several long observations with the Keck telescope and corresponding mean flux densities of Sgr A* are reported in Do et al. (2009) and Hornstein et al. (2007). From their results, after correction for the $L^{\prime}$-extinction used in this work, we obtain an exposure-time-weighted mean de-reddened $L^{\prime}$-flux density of $\mathrm{Sgr} \mathrm{A}^{*}$ of $11.8 \pm 2.6 \mathrm{mJy}$. This is about a factor of 2 higher than the mean emission estimated from the $\mathrm{NaCo} L^{\prime}$ imaging data. However, also the uncertainty of the mean is significantly higher, so that the mean flux density agrees with the $\mathrm{NaCo}$ flux density within about $2 \sigma$. The mean flux density from the Keck observations is based on only 5 epochs or about $8 \mathrm{~h}$ of observing time, while the mean flux from the $\mathrm{NaCo}$ results is based on 19 epochs or roughly $19 \mathrm{~h}$. Therefore the $L^{\prime}$ mean flux density from the Keck results may be biased by a few exceptionally bright events, which is supported by the high standard deviation of the flux densities reported for the Keck observations. 


\subsection{The mean emission from Sgr $A^{*}$ at $2.1 \mu \mathrm{m}$}

The stellar density in the GC is highest near Sgr A* and confusion of the very red counterpart of Sgr A* with stellar sources becomes an increasingly serious problem in the $K$ and $H$ bands (see, e.g., Hornstein et al. 2007), particularly when Sgr A* is in a faint state.

Since it is difficult to disentangle Sgr A* in a low flux state from the faint nearby stars (for a good example, see Fig. 1 in Sabha et al. 2010), work on the variable emission from Sgr A* based on VLT observations was, for a long time, focused almost exclusively on flaring emission. Only recently, Dodds-Eden et al. (2011) presented an extensive and detailed study of the emission of Sgr A* in the $K$ s-band with $\mathrm{NaCo} / \mathrm{VLT}$. Based on a large number of observational epochs, they present a lightcurve with an equivalent length of $\sim 184 \mathrm{~h}$. Although they cannot separate Sgr A* from a stellar source, S17, that is almost coincident with Sgr A* during most epochs, they provide a detailed estimate of the amount of contamination of the emission from $\mathrm{Sgr} \mathrm{A}^{*}$ by stellar light. Their main conclusions relevant to this work are that $\mathrm{Sgr} \mathrm{A} *$ is a continuously variable source in the $K$ s-band and that it spends the overwhelming majority of its time in a low variability state. This low state is termed the quiescent state by the authors and is characterized by a log-normal flux distribution. They derive a de-reddened median flux of $1.6 \mathrm{mJy}$ at the position of of $\mathrm{Sgr} \mathrm{A}^{*}, 0.5 \mathrm{mJy}$ of which is attributed to stellar contamination and $1.1 \mathrm{mJy}$ to $\operatorname{Sgr} \mathrm{A}^{*}$ itself.

At times, Sgr A* can apparently not be detected with $\mathrm{NaCo} / V L T$. For example, Sabha et al. (2010) analyze deep, highquality imaging data from $\mathrm{NaCo}$ during a low phase of Sgr A*. In spite of their imaging data being among the best $K$ s-band GC imaging data obtained at $\mathrm{NaCo} / \mathrm{VLT}$ (in terms of Strehl ratio and stability of AO correction), they do not detect any Sgr A* counterpart and give a de-reddened upper limit of $1-1.5 \mathrm{mJy}$ (extinction corrected for the extinction values assumed in this work). Dodds-Eden et al. (2011) estimate, however, that such extremely low states are relatively rare $(\sim 16 \%$ of the time).

The Keck telescope, on the other hand, is less prone to confusion because of its larger aperture and consequently higher angular resolution. Therefore, work on the emission from Sgr A* based on Keck data has not been biased toward flares or brighter states of Sgr A*. The relevant publications report that $\operatorname{Sgr~A*~is~}$ detected in the $K^{\prime}$-band $(2.12 \mu)$-band at all times (Hornstein et al. 2007; Do et al. 2009; Meyer et al. 2009). Table 1 in Meyer et al. (2009) lists mean $K$-band ${ }^{6}$ flux densities of Sgr A* measured with the Keck telescope during 12 epochs, with one mean flux density from combined Keck/VLT data sets. They also list a mean flux density from one VLT-only observing run. An inspection of the relevant lightcurves in Eckart et al. (2006a) and Meyer et al. (2008) shows that Sgr A* was detected during the cited VLT observations all the time. Here, we exclude the $L^{\prime}$ and the mixed $L^{\prime} / K$-band data sets (first two lines in Table 1 of Meyer et al. 2009). The measurements listed in Meyer et al. (2009) are de-reddened with an extinction of $A_{K}=3.2 \mathrm{mag}$. We removed this reddening correction and calculated the mean flux density of Sgr A* in the $K$-band, weighted by the given length of the observations. It is $0.20 \pm 0.02 \mathrm{mJy}^{7}$, corresponding to a source of $K=16.24 \pm 0.11$. The quality of the NIRC/Keck $K$-data is

\footnotetext{
6 The Keck measurements are in $K^{\prime}$, with a central wavelength of $2.1 \mu \mathrm{m}$, while the VLT measurements are at $K \mathrm{~s}$ with $\lambda_{\mathrm{c}}=2.18 \mu \mathrm{m}$; here, we neglect this difference and assume roughly $\lambda_{\mathrm{c}}=2.1 \mu \mathrm{m}$ also for the two VLT measurements.

7 The unweighted average and standard deviation are $0.208 \pm$ $0.055 \mathrm{mJy}$.
}

high and point-source fitting works well. Also, StarFinder estimates a diffuse background in parallel with point source fitting. This should largely suppress any contamination from unresolved stars, so that a large fraction of the flux measured at the position of Sgr A* will in fact be related to this source, with stellar contamination estimated to be $\$ 35 \%$ by Do et al. (2009). Correction for $A_{K}=2.59 \pm 0.03$ mag of extinction (assuming a central wavelength of $2.1 \mu \mathrm{m}$ and following the extinction law given in Schödel et al. 2010) results in a de-reddened flux density of $2.2 \pm 0.2 \mathrm{mJy}$ at $2.12 \mu \mathrm{m}$ (uncertainty of the extinction correction included).

As can be seen, the situation in the $K$-band is more complicated than in the $L^{\prime}$ - and $M^{\prime}$-bands. Particularly, disentangling Sgr A* from the surrounding stars almost coincident in position with the black hole, requires a very careful analysis and the highest possible angular resolution and image quality. Observing a clearly isolated point source at all times, even in its faintest states, will probably require larger telescope apertures, although the aperture of the Keck telescope appears to be marginally sufficient for this purpose. In any case, the works of Do et al. (2009) and Dodds-Eden et al. (2011) show convincingly that Sgr A* is permanently detectable in the $K$-band with a flux in the range $0.5-2.5 \mathrm{mJy}$.

\section{The IR emission from Sgr $A^{*}$ in the context of its SED}

Work on the infrared emission of $\operatorname{Sgr} \mathrm{A}^{*}$ has so far been focused mainly on the flaring emission, not on the apparently less exciting mean emission. As we have seen, in the sections above, imaging data provide evidence that $\mathrm{SgrA}^{*}$ is detected at 4.8, $3.8 \mu \mathrm{m}$, and $2.1-2.2 \mu \mathrm{m}$ at all times. Moreover, the mean flux of Sgr A* is well defined at these infrared wavelengths. Although the timescales analyzed at the different wavelengths are somewhat different, from a few to $13 \mathrm{~min}$ in case of $M^{\prime}$ and $L^{\prime}$ and subminute timescales in case of $K$, these differences should not be important for the mean SED of Sgr A*, which emphasizes the activity of Sgr A* over long timescales (days to years). Since this paper is focused on the mean NIR/MIR emission from Sgr A*, we are not concerned with the rapid variability of the emission of $\mathrm{Sgr} \mathrm{A}^{*}$ on minute time scales.

Strictly speaking, the mean of a stochastic process (and the flux from Sgr A* can be described in terms of a stochastic process) is only time independent for a (weakly-) stationary process. As the power spectrum of SgrA* follows a broken power-law with a slope for low frequencies consistent with zero and a break timescale of $160 \mathrm{~min}$ (Meyer et al. 2009), it can be expected that the flux's mean is time-independent for averaging times greater than $160 \mathrm{~min}$. This holds true for most of our observations, in particular for the mean flux densities, considered here, which result from an average over a large number of epochs. The analysis in this section implies that there is no significant trend of the mean emission from $\mathrm{Sgr} \mathrm{A}^{*}$ over the time range covered in this work. The $L^{\prime}$ and $M^{\prime}$ data in this work do not provide any evidence for such a trend. Some evidence for variations on time scales of weeks to months is found in the $K$-band flux density measurements by Dodds-Eden et al. (2011). However, so far, there is no evidence for any overall increasing or decreasing long-term trend.

Since it has not been clear for many years whether the infrared counterpart of $\mathrm{Sgr} \mathrm{A}^{*}$ can only be detected during flares and assumes a kind of off-state in between flares, there may still exist some lack of clarity in the community about the term quiescence. Eckart et al. (2006a) referred to a state of "low-level, 
and, especially in the NIR domain, possibly continuously variable flux density" as the interim-quiescent state. Sabha et al. (2010) use the term to refer to a state in which Sgr A* cannot be detected because the images and analysis presented in their work show clearly how difficult, even impossible, it is to detect Sgr A* on $\mathrm{NaCo} / \mathrm{VLT}$ images when it is in a very faint state. Dodds-Eden et al. (2011), however, measured the emission of Sgr A* plus underlying stellar source(s) at the position of Sgr A* in an extensive data set. They estimate in detail the stellar contribution to the flux of Sgr A* in the Ks-band. They define two states by separating the distribution of measured $K$ s-fluxes into a log-normal and a power-law component and refer to the lognormal state of low-variability as the quiescent state.

The distribution of $L^{\prime}$-band fluxes found in this work (see Fig. 7) shows that $\mathrm{Sgr} \mathrm{A}^{*}$ is always clearly detectable at $3.8 \mu \mathrm{m}$, and that it spends much time in a low-variability state, with a tail toward high flux densities, similar to what has been found in the more detailed analysis of Dodds-Eden et al. (2011) for the Ksband. The mean flux density derived for $\mathrm{Sgr}^{*}$ at $L^{\prime}$ is therefore well-defined and probably reflects the quiescent state as defined by Dodds-Eden et al. (2011). Confusion with stellar sources and contamination by stellar flux, respectivly, are of minor importance at $3.8 \mu \mathrm{m}$ as compared to $2.2 \mu \mathrm{m}$ (see, e.g., discussion in Do et al. 2009). This underlines the importance of the $L^{\prime}$ data in the context of the mean SED of Sgr A*.

In the MIR, at a wavelength of $8.6 \mu \mathrm{m}$, we find a de-reddened $3 \sigma$ upper limit of 84 mJy on the quiescent flux density of Sgr A*. This is consistent with, but lower than in previous findings (Schödel et al. 2007) at this wavelength, when differences in extinction estimates and their uncertainties are taken into account. We believe that the improvement is based on the use of imaging data of unprecedented quantity and quality. We take the uncertainty of the extinction correction explicitly into account when estimating the $3 \sigma$ upper limit. Dodds-Eden et al. (2009) report a comparable de-reddened $3 \sigma$ upper limit of $86 \mathrm{mJy}$ at $11.88 \mu \mathrm{m}^{8}$.

We summarize our measurements of the upper limit of the emission at $8.6 \mu \mathrm{m}$ and the mean emission at $3.8 \mu \mathrm{m}$ and $4.8 \mu \mathrm{m}$ of Sgr A* in Table 4, where we also list the mean $K$ band flux densities based on the work of Meyer et al. (2009) and Dodds-Eden et al. (2011). We list the (exposure-time-weighted) uncertainties of the mean flux densities, and not the (exposuretime-weighted) standard deviations because we are focused on the mean SED from Sgr A*. The standard deviations are useful for giving an impression of the epoch-to-epoch variability of Sgr A*. At $3.8 \mu \mathrm{m}(4.8 \mu \mathrm{m})$ they are a factor of $\sim 4(\sim 2.5)$ higher than the uncertainties of the mean flux densities.

Up to now, infrared observations have almost exclusively played a role for modeling the flaring emission from $\mathrm{Sgr}$ A*. For models of the mean emission, the infrared measurements have so far been used only in the form of upper limits. Various models for the SED of Sgr A* are shown in Fig. 8, along with measurements at radio and $\mathrm{X}$-ray wavelengths as well as the new infrared measurements and upper limit from this work. It is clear that the infrared measurements can provide reliable anchor points for the mean SED of Sgr A* on the high-frequency side of the submillimeter bump, providing measurements in a previously existing gap on the order of 6 mag.

The RIAF and the jet-ADAF models shown in Fig. 8 appear to fit the mean infrared fluxes/flux densities and upper limits in

\footnotetext{
8 This is higher than the $57 \mathrm{mJy}$ reported in their work, because they do not explicitly take into account the uncertainty of the extinction correction for their upper limit. The $86 \mathrm{mJy}$ are their $3 \sigma$ upper limit when including the latter source of uncertainty.
}
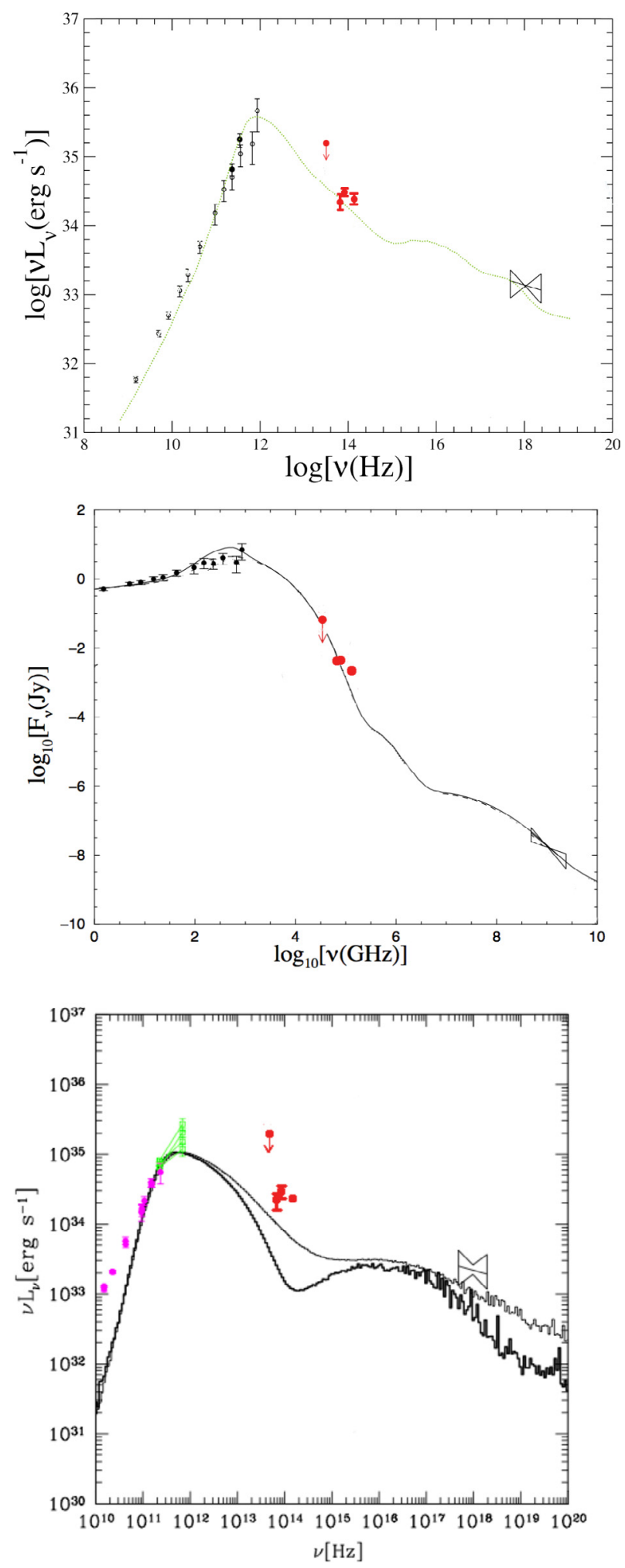

Fig. 8. SED models for the quiescent or mean emission from Sgr A*. Top: RIAF model by Yuan et al. (2004). Middle: jet-ADAF model by Yuan et al. (2002). Bottom: SED from a general relativistic magnetohydrodynamics simulation by Mościbrodzka et al. (2009, their "bestbet" model is shown), where the thin line is the average SED and the thick line represents the SED at a given moment during the simulations. The upper limit at $8.59 \mu \mathrm{m}$ is indicated by a down-pointing red arrow, the mean flux densities at 4.8, 3.8 and $2.1 \mu \mathrm{m}$ are shown as red dots with error bars, which correspond to the standard deviation of the observed variability between epochs. For details on the models and radio/mm/X-ray data, see the referenced publications. 
Table 4. De-reddened mean flux densities of Sgr A* in the infrared.

\begin{tabular}{lcc}
\hline \hline $\begin{array}{l}\lambda \\
(\mu \mathrm{m})\end{array}$ & $\begin{array}{c}f \\
(\mathrm{mJy})\end{array}$ & $\begin{array}{c}\text { Extinction }^{a} \\
(\mathrm{mag})\end{array}$ \\
\hline 8.6 & $84^{b}$ & $2.0 \pm 0.3$ \\
4.8 & $3.8 \pm 1.3$ & $1.0 \pm 0.3$ \\
3.8 & $5.0 \pm 0.6$ & $1.23 \pm 0.08$ \\
$2.1^{c}$ & $2.2 \pm 0.2$ & $2.59 \pm 0.03$ \\
$2.18^{d}$ & 1.1 & 2.5 \\
\hline
\end{tabular}

Notes. ${ }^{(a)}$ Assumed extinction at this wavelength; ${ }^{(b)}$ de-reddened $3 \sigma$ upper limit, including the uncertainty of the extinction correction. The measured $3 \sigma$ upper limit, i.e., not corrected for extinction, is $10 \mathrm{mJy}$; (c) calculated from the data given in Meyer et al. (2009); ${ }^{(d)}$ median flux density from Dodds-Eden et al. (2011). They give a multiplicative standard deviation of 2.1 for the median flux.

general well. The MIR upper-limit lies close to the predicted flux in the jet-ADAF model. The new data points can probably help to fine-tune some parameters of these models.

The GRMHD model from Mościbrodzka et al. (2009) appears to under-predict the IR flux densities. However, we show just one of their many models, i.e., what they call the "best-bet" model. From the models shown in Fig. 4 of their work, it appears that the new infrared measurements generally favor those models (with $T_{i} / T_{\mathrm{e}}=1$ or 3 ) having high angular momentum of the black hole $\left(a_{*} \gtrsim 0.95\right)$ and high inclination angle $(\sim 85 \mathrm{deg})$ of the accretion flow. However, those models appear to become only marginally consistent with the X-ray constraints.

The measured mean emission at 2.1 and $3.8 \mu \mathrm{m}$ and the upper limit at $8.6 \mu \mathrm{m}$ show a clearly increasing trend, in agreement with the models. The $4.8 \mu \mathrm{m}$ point, however, appears to be too low, and not follow the trend. The discrepancy is not major, considering the uncertainties of the measurements. It may be possible that the $M$-band data are biased by insufficient sampling. One has to recall that, due to the low efficiency, the entire $\mathrm{NaCo}$ $M^{\prime}$ data set only comprises a total on-source integration time of just about $650 \mathrm{~s}$. Nevertheless, the mean IR flux densities may also indicate a somewhat flatter slope of the SED in this regime than what is predicted by the models. Therefore this point may merit additional measurements.

\section{On the detectability of Sgr $A^{*}$ in the MIR}

During flares the emission from $\operatorname{Sgr} \mathrm{A}^{*}$ has been reported to rise as high as a few tens of mJy in the NIR (e.g., Dodds-Eden et al. 2009; Kunneriath et al. 2010). However, Sgr A* has so far never been detected in the MIR, not even during flaring activity (Schödel et al. 2007; Dodds-Eden et al. 2009). What can we reasonably expect with VISIR/VLT or similar telescope-instrument combinations?

A considerable amount of observing time has been invested with VISIR/VLT to detect Sgr A*. The sensitivity of VISIR with the PAH1 filter allows the detection of a $5 \mathrm{mJy}$ point source with $10 \sigma$ significance in $1 \mathrm{~h}$ of on-source integration time. These sensitivity estimates were derived from tests on standard stars with chopping and nodding done in a way that the target was always within the detector FOV (see ESO VISIR user's manual). The GC is an extended target, so chopping and nodding have to be done off-source, lowering the observing efficiency to $25 \%$ and thus the $1 \mathrm{~h}$ sensitivity limit by a factor 2 . Due to the limited chop-angle and imperfections on the VISIR detector, some dithering and/or change in chop-angle is also highly recommended (and is usually applied). This will further decrease the observing efficiency. Burst mode observations have an even lower efficiency, about $7 \%$ (estimated from our data), but we judge that, for point sources, the perfect image quality delivered by the holography technique largely offsets the loss in efficiency. Thus, we assume similar sensitivity limits for the burst mode as for the standard observing technique. The theoretical $\sim 10 \mathrm{mJy}$ (at $10 \sigma$ ) limit that can thus be reached under ideal observing conditions in $1 \mathrm{~h}$ of observing time (corresponding to $15 \mathrm{~min}$ on source) is very close to the $3 \sigma$ upper limits derived from our observations. The uncertainty of the upper limit on Sgr A* reported in this work is higher than what could be expected from the theoretical sensitivity, particularly considering the long exposure times of some of the presented images. However, this is because Sgr A* is confused with a ridge of MIR emission and our lack of any image where we know with certainty that the emission from Sgr A* can be neglected, which force us to make conservative assumptions and result in these higher uncertainties.

If we consult the theoretical SED models of Sgr A* that were presented in the previous section, we see that the RIAF model predicts an $8.6 \mu \mathrm{m}$ luminosity about 6 times lower than the current $3 \sigma$ upper limit. The jet model, on the other hand, suggests that $\mathrm{Sgr} \mathrm{A}^{*}$ may linger near the detection limit at MIR wavelengths. Deeper MIR observations may therefore possibly serve to discriminate between pure RIAF and RIAF+jet models. Of course, the models will first have to be updated with their parameters adjusted to the new infrared constraints. Nevertheless, we estimate that the sensitivity of the MIR instrumentation should be increased about five- to ten-fold in order to guarantee significant progress.

Can $\operatorname{SgrA}^{*}$ be detected in the MIR during a flare? The brightest states of the infrared flares, during which detection in the MIR can be expected to be most likely, last normally about $20 \mathrm{~min}$. These are the so-called subflares (e.g., Eckart et al. 2008a). For detecting flares, the time resolution of the MIR observations should be of the same order. Due to the large overheads of MIR observations of the GC, this means, however, that with such a time resolution it is hardly possible to apply dithering and/or to use different chopping positions in order to minimize systematic errors caused by detector defects or the extended flux of the GC region. The data from 22/23 May 2007 should be largely free of such effects, however ${ }^{9}$. From these data we produced five images reconstructed from 4000 different frames each, corresponding to about 19 min time resolution and $80 \mathrm{~s}$ on-source integration for each reconstructed image.

On these images the flux density in a circular aperture of $0.225^{\prime \prime}$ radius was measured in an empty region about $0.5^{\prime \prime}$ NE of Sgr A*. The $1 \sigma$ uncertainty from the measurements was $\sim 7 \mathrm{mJy}$. Additionally, the flux density within an identical aperture was measured on the same images at the position of Sgr A* after subtracting from them the average image reconstructed from all May 2007 data. The inferred $1 \sigma$ uncertainty at the position of Sgr A* was $\sim 5 \mathrm{mJy}$, i.e., close to the one of the empty field (and clearly not larger, as would be expected if Sgr A* had varied). Two of the $20 \mathrm{~min}$ snapshots from the May 2007 data are shown in Fig. 9 in order to give the reader an impression of the random variability of the faint features near Sgr A* on images with such short integration times.

Hence, to detect $\mathrm{Sgr}$ A* with $5 \sigma$ significance during a $20 \mathrm{~min}$ time interval, the source must be on average as bright as $\sim 30 \mathrm{mJy}$ ( $\sim 190 \mathrm{mJy}$ extinction corrected). This is, for example, a factor of

\footnotetext{
9 The imaging data from May 2007 were observed by Schoedel, not downloaded from the archive, i.e. we can be sure of the set-up. Great care was taken to avoid chopping into extended sources.
} 

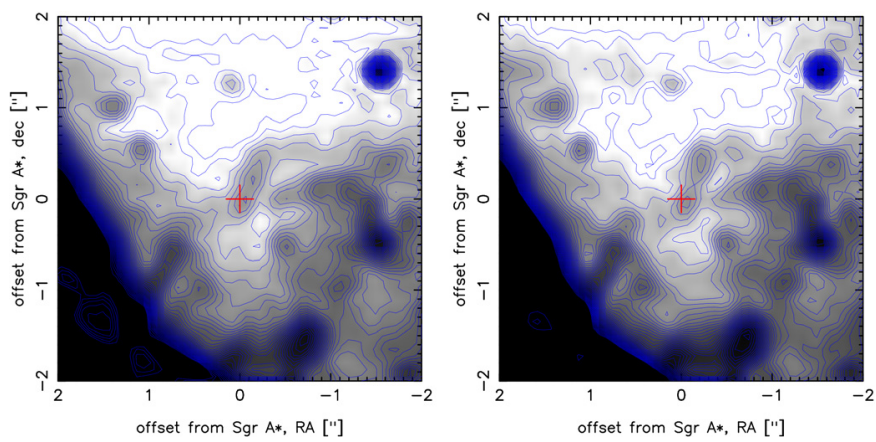

Fig. 9. Two images at $8.6 \mu \mathrm{m}$, reconstructed from subsets of the May 2007 data. Each image is based on a different set of 4000 frames, corresponding to an on-source integration time of $80 \mathrm{~s}$ during an interval of about 19 min. The location of Sgr A* is marked by a red cross. The apparent variability of some of the extended features is caused by the low $S / N$ of these images. The contours are as in Fig. 2.

$>10$ above the quiescent emission predicted by the model shown in the upper panel of Fig. 8.

From NIR measurements it has been inferred that $\operatorname{Sgr} \mathrm{A}^{*}$ has an approximately constant spectral index of $\alpha=-0.6 \pm 0.2$ (for $f \propto v^{\alpha}$, Gillessen et al. 2006; Hornstein et al. 2007, see also Bremer et al. 2011) for flares with de-reddened flux densities $\gtrsim 5 \mathrm{mJy}$ in the $K$-band. Assuming that this spectral index can be extrapolated to the MIR, a flare must be brighter than about $80 \mathrm{mJy}$ (de-reddened) in the $K$-band during at least $20 \mathrm{~min}$ in order to be detected with VLT/VISIR at $8.6 \mu \mathrm{m}$. To the best of our knowledge, such a bright flare has never been reported. The situation would be more favorable if the NIR-toMIR spectral index is steeper, e.g. $\alpha_{K-L}=-1.4 \pm 0.3$ from the mean flux densities at $K^{\prime}$ (Keck data) and $L$ derived here. In that case, it would be sufficient if the flare were brighter than about $24 \mathrm{mJy}$ (de-reddened) in the $K$-band during at least $20 \mathrm{~min}$. Such a steep spectral index is probably an extreme assumption, and such bright flares appear to be extremely rare, which can, for example, be seen clearly in compilations of long time series of the emission from Sgr A* (e.g., Meyer et al. 2008, 2009; Yusef-Zadeh et al. 2009; Dodds-Eden et al. 2011). In fact, this criterion is only met by a single flare reported in the literature (Dodds-Eden et al. 2011).

It may still be possible that $\operatorname{Sgr} \mathrm{A}^{*}$ can be detected in the MIR during exceptional events, such as the brightest $L$-band flare ever reported, during which $\mathrm{Sgr}^{*}$ * showed flux densities between 60 to $90 \mathrm{mJy}$ at $L^{\prime}$ during a full hour (Kunneriath et al. 2010). However, such events appear to be exceptionally rare. Here it is important to note that $\mathrm{Sgr} \mathrm{A}^{*}$ was not even detected in VISIR/VLT observations during the extremely bright, soft-spectrum X-ray flare reported by Porquet et al. (2008, see Dodds-Eden et al. 2009, for the simultaneous MIR observations).

The analysis presented here is for $8.6 \mu \mathrm{m}$. Similar conclusions can be drawn for longer wavelengths, where the increasing thermal background and decreasing angular resolution present additional complications. To summarize, we believe that Sgr A* cannot be detected with VLT/VISIR or similar telescopeinstrument combinations, with the possible exception of rare extreme events. In order to detect $\mathrm{Sgr} \mathrm{A}^{*}$ and examine its variability in the MIR, one needs to combine the highest possible angular resolution with significantly improved detectors. Possibly, very sensitive polarimetric observations could reveal a Sgr A*counterpart during flares (as for the polarization of Sgr A* in the NIR, see, e.g., Eckart et al. 2006b). An alternative may be measurements under the extremely stable and low-background conditions in space. The angular resolution of the JWST will be required for this purpose.

\section{Summary}

This paper presents new observational data on the mean infrared emission from $\mathrm{Sgr} \mathrm{A}^{*}$. No counterpart could be detected at $8.6 \mu \mathrm{m}$, in spite of using an extensive data set and images with excellent Strehl ratios. Detection of a point-source at the location of $\mathrm{Sgr} \mathrm{A}^{*}$ is complicated considerably by the presence of a ridgelike structure, the $\operatorname{Sgr} A^{*}$-Ridge. The $3 \sigma$ upper limit on the flux density from $\mathrm{Sgr} \mathrm{A}^{*}$ at $8.6 \mu \mathrm{m}$ was estimated to be $10 \mathrm{mJy}$ (observed) and $84 \mathrm{mJy}$ after de-reddening. This is lower than what has been previously reported at this wavelength. The upper limit at $8.6 \mu \mathrm{m}$ is mainly dominated by the relatively high and uncertain extinction at this wavelength. Based on the sensitivity of existing imaging data, we argue that MIR emission from Sgr A* - both time-averaged as well as flaring emission - can probably not be detected by imaging observations with current telescopes and instruments. We estimate that about a ten-fold increase in point-source sensitivity is needed in order to provide significant new constraints.

At the shorter wavelengths of $4.8 \mu \mathrm{m}$ and $3.8 \mu \mathrm{m}$, we find from an analysis of an extensive data set that a counterpart of Sgr A* can be detected at all times, and derive its mean emission. Finally, we use/derive the mean emission of $\mathrm{Sgr}^{*}$ at 2.1-2.2 $\mu \mathrm{m}$ from published Keck/VLT data sets, where it has recently been shown to be detectable at all times, too.

The new infrared measurements are in general agreement with current models for the SED of Sgr A*. They do not allow us to clearly distinguish between various published models, but will clearly help to fine-tune the parameters of those models. It should be pointed out that, so far, infrared data have not been included in the models, or, at most, in the form of weak upper limits in the mid-infrared. We can now be sure that $\mathrm{Sgr}^{*}$ is always detectable on the high-frequency side of the Terahertz peak, with a rather well constrained mean flux. The $8.6 \mu \mathrm{m}$ upper limit may be low enough to have an appreciable impact on some models. The data point at $3.8 \mu \mathrm{m}$ is particularly well defined because it is based on a large data set, suffers negligible contamination by stellar light, and the uncertainty of the extinction at this wavelength is low.

Acknowledgements. R.S. acknowledges support by the Ramón y Cajal programme, by grants AYA2010-17631 and and AYA2009-13036 of the Spanish Ministry of Science and Innovation, and by grant P08-TIC-4075 of the Junta de Andalucía. A.A. acknowledges support by grant AYA2009-13036 of the Spanish Ministry of Science and Innovation and by grant P08-TIC-4075 of the Junta de Andalucía. Part of this work was supported by the COST Action MP0905: Black Holes in a violent Universe and PECS project No. 98040.

\section{References}

Baganoff, F. K., Bautz, M. W., Brandt, W. N., et al. 2001, Nature, 413, 45 Balick, B., \& Brown, R. L. 1974, ApJ, 194, 265

Bates, R. H. T., Gough, P. T., \& Napier, P. J. 1973, A\&A, 22, 319

Bremer, M., Witzel, G., Eckart, A., et al. 2011, A\&A, 532, A26

Broderick, A. E., \& Loeb, A. 2006, MNRAS, 367, 905

Christou, J. C. 1991, Exper. Astron., 2, 27

Clénet, Y., Rouan, D., Gratadour, D., et al. 2004, A\&A, 424, L21

Cohen, M., Walker, R. G., Barlow, M. J., \& Deacon, J. R. 1992, AJ, 104, 1650

Diolaiti, E., Bendinelli, O., Bonaccini, D., et al. 2000, in SPIE Conf. Ser., 4007, ed. P. L. Wizinowich, 879

Do, T., Ghez, A. M., Morris, M. R., et al. 2009, ApJ, 691, 1021

Dodds-Eden, K., Porquet, D., Trap, G., et al. 2009, ApJ, 698, 676

Dodds-Eden, K., Gillessen, S., Fritz, T. K., et al. 2011, ApJ, 728, 37 
Doeleman, S. S. Weintroub, J., Rogers, A. E. E., et al. 2008, Nature, 455, 78

Doucet, C., Lagage, P., \& Pantin, E. 2006, in Visions for Infrared Astronomy, Instrumentation, Mesure, Métrologie, ed. V. Coudé du Foresto, D. Rouan, \& G. Rousset, 25

Eckart, A., Baganoff, F. K., Schödel, R., et al. 2006a, A\&A, 450, 535 Eckart, A., Schödel, R., Meyer, L., et al. 2006b, A\&A, 455, 1

Eckart, A., Baganoff, F. K., Zamaninasab, M., et al. 2008a, A\&A, 479, 625

Eckart, A., Schödel, R., García-Marín, M., et al. 2008b, A\&A, 492, 337

Eckart, A., Baganoff, F. K., Morris, M. R., et al. 2009, A\&A, 500, 935

Fritz, T. K., Gillessen, S., Dodds-Eden, K., et al. 2011, ApJ, accepted [arXiv: 1105.2822]

Genzel, R., Schödel, R., Ott, T., et al. 2003, Nature, 425, 934

Genzel, R., Eisenhauer, F., \& Gillessen, S. 2010, Rev. Mod. Phys., 82, 3121

Ghez, A. M., Wright, S. A., Matthews, K., et al. 2004, ApJ, 601, L159

Ghez, A. M., Hornstein, S. D., Lu, J. R., et al. 2005, ApJ, 635, 1087

Ghez, A. M., Salim, S., Weinberg, N. N., et al. 2008, ApJ, 689, 1044

Gillessen, S., Eisenhauer, F., Quataert, E., et al. 2006, ApJ, 640, L163

Gillessen, S., Eisenhauer, F., Trippe, S., et al. 2009, ApJ, 692, 1075

Hornstein, S. D., Matthews, K., Ghez, A. M., et al. 2007, ApJ, 667, 900

Kunneriath, D., Witzel, G., Eckart, A., et al. 2010, A\&A, 517, A46

Lagage, P. O., Pel, J. W., Authier, M., et al. 2004, The Messenger, 117, 12

Lenzen, R., Hartung, M., Brandner, W., et al. 2003, in SPIE Conf. Ser. 4841, ed.

M. Iye, \& A. F. M. Moorwood, 944

Liu, C. Y. C., \& Lohmann, A. W. 1973, Opt. Comm., 8, 372

Lutz, D. 1999, in ESA Spec. Publ., 427, The Universe as Seen by ISO, ed. P. Cox, \& M. Kessler, 623

Lutz, D., Feuchtgruber, H., Genzel, R., et al. 1996, A\&A, 315, L269

Melia, F., \& Falcke, H. 2001, ARA\&A, 39, 309

Meyer, L., Eckart, A., Schödel, R., et al. 2006, A\&A, 460, 15

Meyer, L., Do, T., Ghez, A., et al. 2008, ApJ, 688, L17

Meyer, L., Do, T., Ghez, A., et al. 2009, ApJ, 694, L87

Mezger, P. G., Duschl, W. J., \& Zylka, R. 1996, A\&ARv, 7, 289

Moneti, A., Stolovy, S., Blommaert, J. A. D. L., Figer, D. F., \& Najarro, F. 2001, A\&A, 366, 106
Morris, M., \& Serabyn, E. 1996, ARA\&A, 34, 645

Mościbrodzka, M., Gammie, C. F., Dolence, J. C., Shiokawa, H., \& Leung, P. K. 2009, ApJ, 706, 497

Mužić, K., Eckart, A., Schödel, R., et al. 2010, A\&A, 521, A13

Nishiyama, S., Tamura, M., Hatano, H., et al. 2009, ApJ, 696, 1407

Ott, T., Eckart, A., \& Genzel, R. 1999, ApJ, 523, 248

Paumard, T., Müller, T., Genzel, R., Eisenhauer, F., \& Gillessen, S. 2008, in The Eleventh Marcel Grossmann Meeting On Recent Developments in Theoretical and Experimental General Relativity, Gravitation and Relativistic Field Theories, ed. H. Kleinert, R. T. Jantzen, \& R. Ruffini, 1075

Petr, M. G., Coude Du Foresto, V., Beckwith, S. V. W., Richichi, A., \& McCaughrean, M. J. 1998, ApJ, 500, 825

Porquet, D., Grosso, N., Predehl, P., et al. 2008, A\&A, 488, 549

Quataert, E. 2003, Astron. Nachr. Suppl.. 324, 435

Rafelski, M., Ghez, A. M., Hornstein, S. D., Lu, J. R., \& Morris, M. 2007, ApJ, 659,1241

Rousset, G., Lacombe, F., Puget, P., et al. 2003, in SPIE Conf. Ser., 4839, ed. P. L. Wizinowich, \& D. Bonaccini, 140

Sabha, N., Witzel, G., Eckart, A., et al. 2010, A\&A, 512, A2

Schödel, R., Eckart, A., Mužić, K., et al. 2007, A\&A, 462, L1

Schödel, R., Merritt, D., \& Eckart, A. 2009, A\&A, 502, 91

Schödel, R., Najarro, F., Muzic, K., \& Eckart, A. 2010, A\&A, 511, A18

Stolovy, S. R., Hayward, T. L., \& Herter, T. 1996, ApJ, 470, L45

Tokunaga, A. T., \& Vacca, W. D. 2005, PASP, 117, 1459

Viehmann, T., Eckart, A., Schödel, R., Pott, J., \& Moultaka, J. 2006, ApJ, 642, 861

Yuan, F., Markoff, S., \& Falcke, H. 2002, A\&A, 383, 854

Yuan, F., Quataert, E., \& Narayan, R. 2004, ApJ, 606, 894

Yusef-Zadeh, F., Wardle, M., Heinke, C., et al. 2008, ApJ, 682, 361

Yusef-Zadeh, F., Bushouse, H., Wardle, M., et al. 2009, ApJ, 706, 348

Zamaninasab, M., Eckart, A., Dovčiak, M., et al. 2011, MNRAS, 413, 322 